\title{
Intersection types for explicit substitutions
}

\author{
(Information and Computation, 189(1):17-42, 2004)
}

\author{
Stéphane Lengrand ${ }^{1}$, Pierre Lescanne ${ }^{1}$, Dan Dougherty ${ }^{2}$, Mariangiola \\ Dezani-Ciancaglini ${ }^{\sharp}$, and Steffen van Bakel $^{4}$ \\ 1) École Normale Supérieure de Lyon 46, Allée d'Italie, 69364 Lyon 07, FRANCE, \\ $\left.{ }^{2}\right)$ Department of Computer Science, Worcester Polytechnic Institute Worcester, MA 101609 USA, \\ 3) Dipartimento di Informatica, Università di Torino, Corso Svizzera 185, 10149 Torino, Italy, \\ $\left.{ }^{4}\right)$ Department of Computing, Imperial College of Science, Technology and Medicine, 180 Queen's \\ Gate, London SW7 2BZ, U.K., \\ \{Stephane.Lengrand, Pierre.Lescanne\}@ens-lyon.fr, ddecs.wpi.edu, dezani@di.unito.it, \\ svbedoc.ic.ac.uk
}

\begin{abstract}
We present a new system of intersection types for a composition-free calculus of explicit substitutions with a rule for garbage collection, and show that it characterizes those terms which are strongly normalizing. This system extends previous work on the natural generalization of the classical intersection types system, which characterized head normalization and weak normalization, but was not complete for strong normalization. An important role is played by the notion of available variable in a term, which is a generalization of the classical notion of free variable.
\end{abstract}

keywords: Calculi of explicit substitutions, intersection types, strong normalization.

\section{Introduction}

An explicit substitutions calculus is a refinement of the classical Lambda Calculus (LC) [6] in which substitution is not treated as a meta-operation on terms but rather as an operation of the calculus itself. The inspiration for such a study is the observation that, in the presence of variable-binding, substitution is a complex operation to define and to implement, so that making substitutions explicit leads to a more pertinent analysis of the correctness and efficiency of compilers, theorem provers, and proof-checkers. Abadi, Cardelli, Curien, and Lévy [1] and de Bruijn [12] defined the first calculi of explicit substitutions.

Intersection type disciplines originated in $[14,15]$ to overcome the limitations of Curry's type assignment system and to provide a characterization of the strongly normalizing terms of the $\lambda$-calculus [36]. Since then, intersection types disciplines have been used in a series of papers for characterizing evaluation properties of $\lambda$-terms [29, 28, 3, 4, 23, 2, 22, 17].

As discussed in [20], one can see an explicit substitution calculus as an improvement on both the system of combinators and LC, since it is a system whose mechanics are first-order and as simple as those of combinatory logic, yet which retains the same intensional character as LC. Observe that LC can be viewed as a subsystem of explicit substitution systems, defined by the strategy of "eagerly" applying the substitution induced by contracting a $\beta$-redex. In this sense,

\footnotetext{
${ }^{\dagger}$ Partially supported by EU within the FET - Global Computing initiative, Project DART ST-2001-33477, and MURST Projects COMETA and McTati. The funding bodies are not responsible for any use that might be made of the results presented here.
} 
explicit substitutions calculi are logically prior to LC, and the study of explicit substitutions represents a deeper examination of the relationship between abstraction and application.

A fundamental property of typed LC is strong normalization: no typable term admits an infinite reduction sequence. Melliès [33] made the somewhat surprising discovery that strong normalization fails even for simply-typed terms of the Abadi-Cardelli-Curien-Lévy calculus.

Given the central place that strong normalization occupies in the theory and application of LC, it is important to study this property in systems of explicit substitutions. Melliés' result exploits the existence of a composition operator on substitutions, and so there are two obvious and complementary directions for research. The first is to define classes of reduction strategies in the original calculus which support strong normalization; a notable example of work in this area is that of Eike Ritter [37]. The second direction is to investigate calculi in which substitutions are explicit but composition is absent; the current paper is part of this effort.

Composition-free calculi of explicit substitutions have been studied in $[31,11,26,10,8]$, among other works. Here we work in the composition-free calculus $\lambda \mathrm{x}$ [11] and a calculus $\lambda \mathrm{x}_{\mathrm{gc}}$ obtained by adding explicit garbage collection to $\lambda \mathrm{x}$. In fact, our rule for garbage collection is stronger than the one originally presented in [11].

Previous work $[19,20]$ explored some reduction properties of this system using intersection types. The natural generalizations of the classical type systems were able to characterize the sets of normalizing and head-normalizing terms by means of typability. But it was shown in [19] that the naive generalization of the classical system did not characterize the strongly normalizing terms. Typable terms were strongly normalizing but the converse fails.

Example 1.1 Consider the terms

$$
\begin{aligned}
& M_{1} \equiv((\lambda y . z)(x x))\langle x=\lambda a . a a\rangle \text { and } \\
& M_{2} \equiv z\langle y=x x\rangle\langle x=\lambda a . a a\rangle
\end{aligned}
$$

and notice that $M_{1} \longrightarrow M_{2}$. The term $M_{2}$ is readily seen to be strongly normalizing. But $M_{2}$ is not typable in the system $\mathcal{D}$ of [19]: it is obtained from the (not strongly normalizing, hence untypable) term $M_{1}$ by contracting a $\beta$-redex, and such a contraction does not change the typing behaviour of terms under $\mathcal{D}$. Finding a type system characterizing the strongly normalizing terms was left as an open problem in [19].

Main results. In this paper we solve the aforementioned problem: we define an extension $\mathcal{E}$ of system $\mathcal{D}$ which types precisely the strongly normalizing terms. Furthermore, when a universal type $\omega$ is added, the resulting system $\mathcal{E}_{\omega}$ satisfies the same theorems as those in [19] characterizing the weakly normalizing, head normalizing, and solvable terms. Our claim, then, is that the system presented here - with or without a universal type - is a robust type system appropriate for analyzing reduction properties in explicit substitutions calculi.

In fact, we present two different characterizations of strong normalization, in the form of two different type systems. These systems were discovered independently $[5,30]$. Each system starts with the natural generalization of the classical intersection types system to the explicit substitutions calculus and adds a new typing rule. In one system [5], the new rule essentially takes into account that, by putting a term of the shape $M\langle x=N\rangle$ - where $x$ does not occur free in $M-$ in an arbitrary context, the free variables of $N$ will never be replaced. Therefore, we can discharge the assumptions used to type $N$ when we derive a type for $M\langle x=N\rangle$. For the second system the key insight for the solution is a new notion, that of available variable occurrence in a term (Definition 2.2). This is a refinement of the notion of free variable.

The present paper is an joint expanded version of the conference papers [5] and [30]; we 
present both rules in a uniform system, and investigate the relationship between the two systems.

As a corollary of our proof methods we are able to define a somewhat more general notion of garbage collection than has been studied in the literature of $\lambda \mathrm{x}$ and show that adding a reduction for garbage collection does not change the set of strongly normalizing terms.

Explicit substitutions calculi without composition typically enjoy the preservation of strong normalization property: a pure term is strongly normalizing in the presence of explicit substitutions if it is so under $\beta$-reduction $[32,8,11,9,10,38,18]$. It follows that the classical intersection type system does characterize strong normalization for pure terms. In contrast, the current results provide information about all terms. Perhaps more significant is the fact that the proofs here are direct, involving reasoning in the explicit substitutions calculus itself, not passing through the indirection of an argument about $\beta$-reduction. Herbelin [25] has proposed also a direct proof of strong normalization for a simply typed calculus of explicit substitution which interprets a sequent calculus (he restricts the attention to simple types and so does not achieve a characterization of strong normalization). We recommend his introduction for other arguments on how explicit substitutions give an account of the cut rule [21].

Recently we learned that Jean Goubault-Larrecq proposes, in the exercises of his course [24], a type system with intersection types for (a version with De Bruijn indices of) the calculus of explicit substitutions $\lambda v$ introduced in [31]. Each typable term in this calculus is shown to be strongly normalizing, but the converse is not true.

Plan of the paper. Section 2 presents the syntax and reduction semantics of $\lambda x$, and in Section 3 we derive some important technical results about reduction, including the definition of a perpetual strategy and an inductive definition of the set of strongly normalizing terms. In Section 4 we present the type system $\mathcal{E}$ and we show the inter-admissibility of the two new typing rules we define. In Section 5 we prove that all strongly normalizing terms are typable in system $\mathcal{E}$, and in Section 6 we show the converse. Finally, in Section 7, we verify that the results of [19] extend to system $\mathcal{E}_{\omega}$.

Notation. Our notation is consistent with that of [7], to which we refer the reader for background on LC. We will use $\underline{n}$ for $\{1, \ldots, n\}$.

\section{The calculus $\lambda \mathrm{x}$}

\subsection{Syntax and available variables}

Definition 2.1 The set $\lambda \mathrm{x}$ of terms with explicit substitutions is defined as follows :

$$
M, N::=x|\lambda x \cdot M| M N \mid M\langle x=N\rangle
$$

A term of the form $M\langle x=N\rangle$ is called a closure. A term which contains no closure is called a pure term.

In writing terms, we will use the standard conventions for removing brackets, and use the following abbreviations:

$$
\begin{aligned}
\vec{M} & =M_{1}, \ldots, M_{n}(n \geq 0) \\
M \vec{M} & =M M_{1} \ldots M_{n}(n \geq 0) \\
M \overline{\langle x=N\rangle} & =M\left\langle x_{1}=N_{1}\right\rangle \ldots\left\langle x_{n}=N_{n}\right\rangle(n \geq 0)
\end{aligned}
$$

We will see in Figure 2 another description of the set of terms with explicit substitutions called the head-form taxonomy whereas the above description could be called the natural tax- 
onomy. One defines the notions of free and bound variable occurrences in a term as usual. But it turns out that in the presence of explicit substitutions a refinement of the notion of free variable, called available variable occurrence, is key.

Definition 2.2 The free variables in a term are:

$$
\begin{array}{ll}
f_{v}(x) & =\{x\} \\
f v(\lambda x . M) & =f v(M) \backslash\{x\} \\
f v(M N) & =f v(M) \cup f v(N) \\
f v(M\langle x=N\rangle) & =(f v(M) \backslash\{x\}) \cup f_{v}(N)
\end{array}
$$

A variable occurrence which is not free is called a bound occurrence. The available variables in a term are:

$$
\begin{array}{ll}
a v(x) & =\{x\} \\
a v(\lambda x . M) & =a v(M) \backslash\{x\} \\
a v(M N) & =a v(M) \cup a v(N) \\
a v(M\langle x=N\rangle) & = \begin{cases}a v(N) \cup a v(M) \backslash\{x\}, & \text { if } x \in a v(M) \\
a v(M), & \text { if } x \notin a v(M)\end{cases}
\end{array}
$$

It is easy to show by induction on the structure of terms that the available variable occurrences in a term are a subset of the free variable occurrences, and that free and available variables coincide for pure terms.

Lemma 2.3 av $(M) \subseteq f v(M)$.

Availability differs from freeness in that the available variables of $M\langle x=N\rangle$, where $x$ is not available in $M$, are exactly those of $M$, whereas the free variables in any case are those of $M$ and $N$. The intuition is that $x$ is not available just when the term $N$ disappears in the course of fully applying the substitutions in $M\langle x=N\rangle$.

Further discussion of the motivation for defining available variable occurrences will be given after we present our type system. For now we can observe, referring to Example 1.1, that, in the term $z\langle y=x x\rangle$, the variable $x$ is free, but not available.

Notice that, actually, the calculus includes two binders, namely $\lambda$ in $\lambda x$.M which binds $x$ in $M$, and also $\cdot\langle\cdot=\cdot\rangle$ in $M\langle x=N\rangle$ which binds $x$ in $M$. In what follows, we consider terms up to $\alpha$-conversion. Throughout this paper, we will assume the Barendregt convention on variables [6] to be fulfilled: no variable occurs both free and bound. Since available variables are free it follows that we assume that no variable occurs both available and bound in the same context. The Barendregt convention extends to judgments $\Gamma \vdash M: \sigma$ (see Definition 4.5) in which variables occurring in the judgment $\Gamma$ are considered as free and cannot occur bound in the term $M$. Thus a judgment like $(x: \sigma) \vdash M\langle x=N\rangle: \tau$ is prohibited by the Barendregt convention. 


\subsection{The rules}

Definition 2.4 ( $\lambda \mathrm{x}$ AND $\left.\lambda \mathrm{x}_{\mathrm{gc}}\right)$ We identify the following reduction rules on $\lambda \mathrm{x}$ terms.

$$
\begin{array}{lll}
(\lambda x \cdot M) P & \longrightarrow M\langle x=P\rangle & (\mathrm{B}) \\
(M N)\langle x=P\rangle & \longrightarrow M\langle x=P\rangle N\langle x=P\rangle & (\text { App) } \\
(\lambda y \cdot M)\langle x=P\rangle & \longrightarrow \lambda y \cdot(M\langle x=P\rangle) & \text { (Abs) } \\
x\langle x=P\rangle & \longrightarrow P & \text { (Varl) } \\
y\langle x=P\rangle & \longrightarrow y & \text { (Vark) } \\
M\langle x=P\rangle & \longrightarrow M \text {, if } x \notin a v(M) & \text { (gc) }
\end{array}
$$

The Barendregt convention on variables plays a major role in the above definition, especially in rule (Abs) which otherwise would involve the capture of variables. The notion of reduction $\lambda \mathrm{x}$ is obtained by deleting rule (gc), and the notion of reduction $\lambda \mathrm{x}_{\mathrm{gc}}$ is obtained by deleting rule (VarK). The rule (gc) is called "garbage collection", as it removes useless substitutions. Notice that here we propose a form of the (gc) rule which differs from the similar rules given in $[11,20]$, in that it uses availability of the variable instead of freeness. This models a more liberal rule for garbage collection.

When it is clear from the context which notion of reduction is used, $\longrightarrow$ will denote the reduction relation and $\longrightarrow$ will denote its reflexive and transitive closure.

We will now formally justify the addition or our rule (gc) to $\lambda \mathrm{x}$.

Lemma 2.5 Let $M={ }_{\lambda \mathrm{x}} N$ be the equivalence relation on terms generated by the rules of $\lambda \mathrm{x}$.

i) $(M\langle y=Q\rangle)\langle x=N\rangle={ }_{\lambda \mathbf{x}}(M\langle x=N\rangle)\langle y=Q\langle x=N\rangle\rangle$

ii) $M\langle x=N\rangle=\lambda_{\mathrm{x}} M$, if $x \notin a v(M)$.

Proof: i) By induction on the structure of terms, using the fact that $\longrightarrow_{\lambda \mathrm{x}}$ is weakly normalizing (see [38], page 71, Lemma 3.1.12).

ii) By induction on the structure of terms. Cases $M \equiv y, \lambda y . P, P Q$ are straightforward (indeed, for those even ' $\longrightarrow \lambda_{\mathrm{x}}{ }^{\prime}$ could be used). For the remaining case, $M \equiv P\langle y=Q\rangle$, first of all, notice that, we have $(P\langle y=Q\rangle)\langle x=N\rangle={ }_{\lambda \mathrm{x}}(P\langle x=N\rangle)\langle y=Q\langle x=N\rangle\rangle$ by the previous part. We distinguish two cases:

$-y \in a v(P)$. Then $x \notin a v(Q) \cup a v(P) \backslash\{y\}$, so

$$
\begin{array}{ll}
(P\langle x=N\rangle)\langle y=Q\langle x=N\rangle\rangle & ={ }_{\lambda \mathrm{x}}(I H) \\
P\langle y=Q\langle x=N\rangle\rangle & ={ }_{\lambda \mathrm{x}}(I H) \quad P\langle y=Q\rangle .
\end{array}
$$

- $y \notin a v(P)$; notice that then also $P\langle y=Q\rangle={ }_{\lambda \mathrm{x}} P$, by induction. Then $x \notin a v(P)$, so

$$
\begin{array}{ll}
(P\langle x=N\rangle)\langle y=Q\langle x=N\rangle\rangle & ={ }_{\lambda \mathbf{x}}(I H) \\
P\langle y=Q\langle x=N\rangle\rangle & =\lambda_{\mathbf{x}}(I H) \quad P
\end{array}
$$

So $(P\langle x=N\rangle)\langle y=Q\langle x=N\rangle\rangle={ }_{\lambda \mathbf{x}} P\langle y=Q\rangle$.

In particular, for both cases, we get $(P\langle y=Q\rangle)\langle x=N\rangle={ }_{\lambda \mathbf{x}} P\langle y=Q\rangle$.

By induction on reductions one can check that the set of available variables does not increase when terms are reduced.

Lemma 2.6 i) If $M \longrightarrow N$ then av $(M) \supseteq$ av $(N)$. 


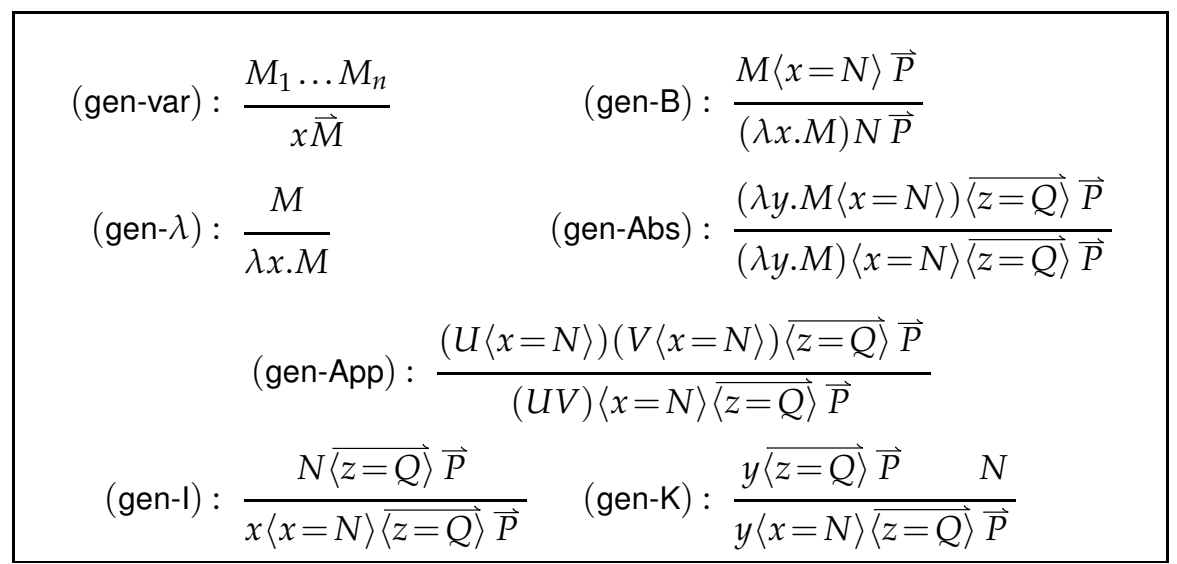

Figure 1: Generation of $\mathcal{S N}$

ii) If $x \notin a v(M), M \longrightarrow N$ and $N$ is a pure term then $x \notin f v(N)$.

In contrast with LC we are considering a rewrite system with several rules, which in fact interact with each other in interesting ways. For example, there is a critical pair formed by the rules (B) and (App). Specifically, a term of the form

$$
((\lambda x . M) N)\langle y=L\rangle
$$

can be reduced to either of

$$
(\lambda x . M)\langle y=L\rangle N\langle y=L\rangle \quad \text { or } \quad M\langle x=N\rangle\langle y=L\rangle
$$

Most of the difficulty in working with the system is due to this critical pair, as we will see.

Definition $2.7(\mathcal{S N})$ We say, as usual, that $M$ is in normal form if $M$ is redex free, and write $n f(M)$ if $M$ is in normal form. $M$ is normalisable is there exists $M^{\prime}$ in normal form such that $M \longrightarrow M^{\prime}$, and $M$ is strongly normalisable if all reduction sequences starting in $M$ are of finite length. We use $\mathcal{S N}$ for the set of strongly normalizing terms under $\lambda \mathrm{x}$.

\section{Generation of $\mathcal{S N}$, saturated sets, and a perpetual strategy}

In this section we show some properties of the set $\mathcal{S N}$ : the only property which is needed for our characterization result is that $\mathcal{S N}$ is saturated (Theorem 3.4), but we think that the perpetuality of the defined strategy is by itself interesting.

\subsection{An inductive characterization of $\mathcal{S N}$}

We first recall a key closure condition of $\mathcal{S N}$ proved in [20].

Lemma 3.1 The set $\mathcal{S N}$ is closed under rule:

$$
\text { (subs) : } \frac{M\langle y=L\rangle\langle x=N\langle y=L\rangle\rangle\langle\overrightarrow{\langle z=Q\rangle} \vec{P}}{M\langle x=N\rangle\langle y=L\rangle \overrightarrow{\langle z=Q\rangle \vec{P}}}
$$

Figure 1 tells us how the set of strongly normalizing terms can be generated by induction. Rule (gen-var) has a number (possibly zero) of terms as upper part. The rule (gen-App) is 
interesting, as a first example of the role of our critical pair. When the term $(U V)$ is in fact a B-redex, it is not obvious that this rule is sound, that is, that pushing the substitution through the application (as opposed to firing the B-redex) preserves the existence of an infinite reduction. But in fact it is sound, as we will see.

Property $3.2 \mathcal{S N}$ is generated by the rules of Figure 1.

Proof: We first show that the rules in Figure 1 generate only terms in $\mathcal{S N}$, i.e. that for each rule, if the upper term(s) belong to $\mathcal{S N}$ then the lower term belong to $\mathcal{S N}$.

We only consider two of the rules: (gen-I), because it is typical, and (gen-App), because it uses techniques specific to this set of rules.

(gen-I) : Suppose $N \overrightarrow{\langle z=Q\rangle} \vec{P}$ is in $\mathcal{S N}$. Suppose, towards a contradiction, that $x\langle x=N\rangle\langle z=Q\rangle P$ is not in $\mathcal{S N}$, then there is an infinite reduction starting from this term. Either

- this reduction never contracts the left-outermost redex $x\langle x=N\rangle$ and there exists an infinite reduction starting from $N$ or one of the $Q_{i}{ }^{\prime}$ s or one of the $P_{j}{ }^{\prime}$ s, then $N \overrightarrow{\langle z=Q\rangle} \vec{P}$ is not in $\mathcal{S N}$, which is a contradiction.

- or this reduction is of the form

$$
\begin{aligned}
x\langle x=N\rangle \overline{\langle z=Q\rangle} \vec{P} & \longrightarrow x\left\langle x=N^{\prime}\right\rangle \overline{\left\langle z=Q^{\prime}\right\rangle} \vec{P}^{\prime} \\
& \longrightarrow N^{\prime} \overline{\left\langle z=Q^{\prime}\right\rangle \vec{P}^{\prime}} \\
& \longrightarrow
\end{aligned}
$$

which is in contradiction with the fact that $N \overrightarrow{\langle z=Q\rangle} \vec{P} \in \mathcal{S N}$.

(gen-App): Suppose $(U V)\langle x=N\rangle\langle\overrightarrow{z z=Q\rangle} P$ is not in $\mathcal{S N}$, then there exists an infinite reduction starting from this term. If this reduction never reduces the redex $(U V)\langle x=N\rangle$, neither by (App), nor by (B) (in which case $U$ reduces to an abstraction), then there exists an infinite reduction starting from $U$, or $V$, or $N$ or one of the $Q_{i}$ 's or one of the $P_{j}$ 's, and $(U\langle x=N\rangle)(V\langle x=N\rangle) \overline{\langle z=Q\rangle}$ is not in $\mathcal{S N}$, which is a contradiction. If

$$
\begin{aligned}
(U V)\langle x=N\rangle \overline{\langle z=Q\rangle} \vec{P} & \longrightarrow\left(U^{\prime} V^{\prime}\right)\left\langle x=N^{\prime}\right\rangle \overline{\left\langle z=Q^{\prime}\right\rangle} \vec{P}^{\prime} \\
& \longrightarrow\left(U^{\prime}\left\langle x=N^{\prime}\right\rangle\right)\left(V^{\prime}\left\langle x=N^{\prime}\right\rangle\right) \overline{\left\langle z=Q^{\prime}\right\rangle \vec{P}^{\prime}}
\end{aligned}
$$

then the looked for contradiction comes from the fact that we have assumed that $(U\langle x=N\rangle)(V\langle x=N\rangle) \overrightarrow{\langle z=Q\rangle} \vec{P}$ is in $\mathcal{S N}$. Suppose now that

$$
\begin{aligned}
& (U V)\langle x=N\rangle \overline{\langle z=Q\rangle} \vec{P} \rightarrow\left(\left(\lambda y \cdot U^{\prime}\right) V^{\prime}\right)\left\langle x=N^{\prime}\right\rangle \overline{\left\langle z=Q^{\prime}\right\rangle} \vec{P}^{\prime} \\
& \longrightarrow\left(U^{\prime}\left\langle y=V^{\prime}\right\rangle\left\langle x=N^{\prime}\right\rangle\right) \overline{\left\langle z=Q^{\prime}\right\rangle} \vec{P}^{\prime} \text {. }
\end{aligned}
$$

But the assumption is that $(U\langle x=N\rangle)(V\langle x=N\rangle) \overrightarrow{\langle z=Q\rangle} \vec{P}$ is in $\mathcal{S N}$, so also $U^{\prime}\left\langle x=N^{\prime}\right\rangle\left\langle y=V^{\prime}\left\langle x=N^{\prime}\right\rangle\right\rangle\left\langle\overline{\left\langle z=Q^{\prime}\right\rangle} \vec{P}^{\prime}\right.$ is in $\mathcal{S N}$. Therefore, by (Lemma 3.1), applying (subs) gives that $\left(U^{\prime}\left\langle y=V^{\prime}\right\rangle\left\langle x=N^{\prime}\right\rangle\right) \overline{\left\langle z=Q^{\prime}\right\rangle} \vec{P}^{\prime}$ in $\mathcal{S N}$, which is a contradiction.

To conclude the proof, we need to show that the rules in Figure 1 generate all the terms in $\mathcal{S N}$. This is proven by a double induction on the length of the longest derivation to normal form and on the structure of terms. Notice that the terms in the conclusions of the given rules cover all possible shapes of terms in $\lambda \mathrm{x}$. Moreover, it is easy to see that for each rule in Figure 1, if the lower term belong to $\mathcal{S N}$ then the upper term(s) belong to $\mathcal{S N}$. The induction hypothesis applies since the the upper term(s) either can be obtained by reducing the lower term or they are subterms of the lower term. 


\subsection{Saturated sets}

In order to define the notion of saturated set we identify a new closure-condition on sets of terms.

$$
\text { (gen-gc) } \frac{M \overline{\langle z=Q\rangle} \vec{P} \quad N \in \mathcal{S N}}{M\langle x=N\rangle \overline{\langle z=Q\rangle} \vec{P}}(x \notin a v(M))
$$

Definition 3.3 A set closed under the rules (subs), (gen-B), (gen-Abs), (gen-App), (gen-I) and (gen-gc) is said to be $\mathcal{S N}$-saturated.

Theorem 3.4 (SATURATion of $\mathcal{S N}$ ) The set $\mathcal{S N}$ is $\mathcal{S N}$-saturated.

Proof: Because of Lemma 3.1 and Proposition 3.2 we need only to show that $\mathcal{S N}$ is closed under the new rule. To show closure under rule (gen-gc), we reformulate the proof of [20] to take into account the change from $f v(\cdot)$ to $a v(\cdot)$ in the definition of $\lambda \mathrm{x}_{\mathrm{gc}}$. We define an $n-$ multi-context as a term with $n$ holes in which we can insert $n$ terms, or simply multi-context if $n$ is understood from the context. If $C[[\cdot, \ldots, \cdot]]$ is an $n$-multi-context and $M_{1}, \ldots, M_{n}$ are terms, then the insertions of those terms in $C[[\cdot, \ldots, \cdot]]$ is denoted $C\left[\left[M_{1}, \ldots, M_{n}\right]\right]$, or $C\left[\left[\vec{M}_{i}\right]\right.$ for short. We prove the following more general statement:

Let $C[[.]$.$] be a multi-context, and N_{i}, M_{i}, i \in \underline{n}$ be terms, with $x \notin$ av $\left(M_{i}\right)$, for $i \in \underline{n}$. If $C\left[\left[M_{i}\right]\right] \in$ $\mathcal{S N}$ and $N_{i} \in \mathcal{S N}$ for $i \in \underline{n}$ then $\left.C\left[\overline{M_{i}\left\langle x=N_{i}\right\rangle}\right]\right] \in \mathcal{S N}$.

We consider triples $\langle P, \boldsymbol{M}, \boldsymbol{N}\rangle$, where $P$ is a term, $\boldsymbol{M}$ and $N$ are multisets of terms. Let $\sqsupset^{m}$ be the multiset extension [16] of $\sqsupset$, the converse of the proper subterm order, and let $\longrightarrow^{m}$ be the multiset extension of the reduction relation $\lambda \mathrm{x}_{\mathrm{gc}}$. The proof is by induction over the following relation:

$\langle P, \boldsymbol{M}, \boldsymbol{N}\rangle \gg\left\langle P^{\prime}, \boldsymbol{M}^{\prime}, \boldsymbol{N}^{\prime}\right\rangle$ if and only if

$$
\begin{array}{rll}
P & \longrightarrow & P^{\prime} \\
P=P^{\prime} \text { or } \\
\boldsymbol{M} & \sqsupset^{m} & \boldsymbol{M}^{\prime} \text {, or } \\
P=P^{\prime}, \boldsymbol{M}=\boldsymbol{M}^{\prime} \text {, and } \boldsymbol{N} & \longrightarrow^{m} & \boldsymbol{N}^{\prime} .
\end{array}
$$

In what follows, $P$ will be $C\left[\llbracket M_{i}\right]$ and $\longrightarrow$ is well-founded out of $P$ by hypothesis; $M$ will be $\left\{M_{1}, \ldots, M_{n}\right\} ; N$ will be $\left\{N_{1}, \ldots, N_{n}\right\}$ and its $\lambda \mathrm{x}_{\mathrm{gc}}$-reducts. The relation $\longrightarrow^{m}$ will be wellfounded since multiset extension preserves well-foundedness. Therefore, $\gg$ is well-founded and a Nötherian induction on $\gg$ is possible.

A remark on cases $((i v))$ and $((v))$ below: there the term $P$ does not change, only its representation as $C[[\ldots]]$ does. This means we insert the $N_{i}$ 's at "lower" positions, allowing us to perform a Nötherian induction.

Assume that $C\left[\left[\overrightarrow{M_{i}} \rrbracket \in \mathcal{S N}\right.\right.$, and that $N_{i} \in \mathcal{S N}$ for $i \in \underline{n}$. We will prove that the term $C\left[\llbracket \overrightarrow{M_{i}}\left\langle x=N_{i}\right\rangle\right]$ reduces only to terms that are in $\mathcal{S N}$.

i) $\left.C\left[\left[\overrightarrow{M_{i}\left\langle x=N_{i}\right\rangle}\right]\right] \longrightarrow C^{\prime}\left[\overrightarrow{M_{i_{j}}\left\langle x=N_{i_{j}}\right\rangle}\right]\right]$ (where the $i_{j} \in \underline{n}$ ):

Then $C\left[\left[\overrightarrow{M_{i}}\right]\right] \longrightarrow C^{\prime}\left[\left[\overrightarrow{M_{i_{j}}}\right]\right.$, and by induction $C^{\prime}\left[\left[\overrightarrow{M_{i_{j}}}\left\langle x=N_{i_{j}}\right\rangle\right] \in \mathcal{S N}\right.$.

ii) $M_{i} \longrightarrow M_{i}^{\prime}$ : By induction.

iii) $N_{j} \longrightarrow N_{j}^{\prime}$ : Also by induction. Note that this case occurs only when the $N_{i}$ are in $\mathcal{S N}$.

iv) $M_{i}=M_{i}^{1} M_{i}^{2}$ and $M_{i}\left\langle x=N_{i}\right\rangle \longrightarrow M_{i}^{1}\left\langle x=N_{i}\right\rangle M_{i}^{2}\left\langle x=N_{i}\right\rangle$ : Since

$$
\left\{M_{1}, \ldots, M_{i}, \ldots, M_{n}\right\} \sqsupset^{m} \quad\left\{M_{1}, \ldots, M_{i}^{1}, M_{i}^{2}, \ldots, M_{n}\right\},
$$


we have $C\left[\left[M_{1}\left\langle x=N_{1}\right\rangle, \ldots,\left(M_{i}^{1}\left\langle x=N_{i}\right\rangle M_{i}^{2}\left\langle x=N_{i}\right\rangle\right), \ldots, M_{n}\left\langle x=N_{n}\right\rangle\right] \in \mathcal{S N}\right.$ by induction.

v) $M_{i}=\lambda y \cdot M_{i}^{\prime}$ and $M_{i}\left\langle x=N_{i}\right\rangle \longrightarrow \lambda y \cdot\left(M_{i}^{\prime}\left\langle x=N_{i}\right\rangle\right)$ :

$$
\left\{M_{1}, \ldots, M_{i}, \ldots, M_{n}\right\} \sqsupset^{m}\left\{M_{1}, \ldots, M_{i}^{\prime}, \ldots, M_{n}\right\},
$$

hence $C\left[\llbracket M_{1}\left\langle x=N_{1}\right\rangle, \ldots, \lambda y \cdot\left(M_{i}^{\prime}\left\langle x=N_{i}\right\rangle\right), \ldots, M_{n}\left\langle x=N_{n}\right\rangle\right] \in \mathcal{S N}$ by induction.

vi) $M_{i}\left\langle x=N_{i}\right\rangle \longrightarrow M_{i}$, which is always applicable being $x \notin a v\left(M_{i}\right)$ : Since

$$
\left\{M_{1}, \ldots, M_{i-1}, M_{i}, M_{i+1}, \ldots, M_{n}\right\} \sqsupset^{m} \quad\left\{M_{1}, \ldots, M_{i-1}, M_{i+1}, \ldots, M_{n}\right\},
$$

also $C\left[\left[M_{1}\left\langle x=N_{1}\right\rangle, \ldots, M_{i}, \ldots, M_{n}\left\langle x=N_{n}\right\rangle\right]\right] \in \mathcal{S N}$ by induction.

We have shown that $\mathcal{S N}$ is closed under the rule (gen-gc). This has as a consequence that $\mathcal{S N}$ is also the set of terms strongly normalizing under $\lambda \mathrm{x}_{\mathrm{gc}}$.

\subsection{A perpetual strategy}

In what follows we will define a perpetual strategy for our calculus, which is an extension to $\lambda \mathrm{x}$ of the strategy defined in [6], page 338. It is based on the reduction of perpetual redexes.

Definition 3.5 (Perpetual Redex) For any term not in normal form, we define its perpetual redex.

- The perpetual redex of $\lambda x . M$ is the perpetual redex of $M$.

- The perpetual redex of $M N$ is :

$M N$, $\quad$ if $M N$ itself is a redex

the perpetual redex of $M$, if $M$ is not a normal form

the perpetual redex of $N$, otherwise

- The perpetual redex of $M\langle x=N\rangle$ is :

the perpetual redex of $N$, if $M \equiv y \neq x$ and $N$ is not a normal form

the perpetual redex of $M$, if $M$ is a closure

$M\langle x=N\rangle$, otherwise

Definition 3.6 (Perpetual StRATEgY) The perpetual strategy is the strategy that reduces always the perpetual redex. It is denoted by $\rightsquigarrow$.

Figure 2 gives both the perpetual strategy and a partition of terms according to the head-form taxonomy. The right-hand sides of rules (perp- $\lambda$ ) and (perp-var) give two forms of irreducible terms when $n f(M)$ and $\vec{Q}$ is empty. Then together with the left-hand sides of the other rules they split the set of terms into classes that form the head-normal form taxonomy.

Since each term contains at most one perpetual redex, the perpetual strategy is deterministic. Note that, in the case of $\lambda \mathrm{x}_{\mathrm{gc}}$, the perpetual strategy never reduces by (gc), except when (gc) is degenerated into (Vark), which means that in this case the perpetual redex is of the form $y\langle x=N\rangle$.

The perpetual strategy is intended to terminate on a term only when the term is strongly normalizing. This is why it does not reduce a term $y\langle x=N\rangle$ by (Vark) or (gc) when $N$ is not a normal form. Indeed, if $N$ is not strongly normalizing, the perpetual strategy (to be really perpetual) has to reduce $N$ instead of causing it to disappear. 


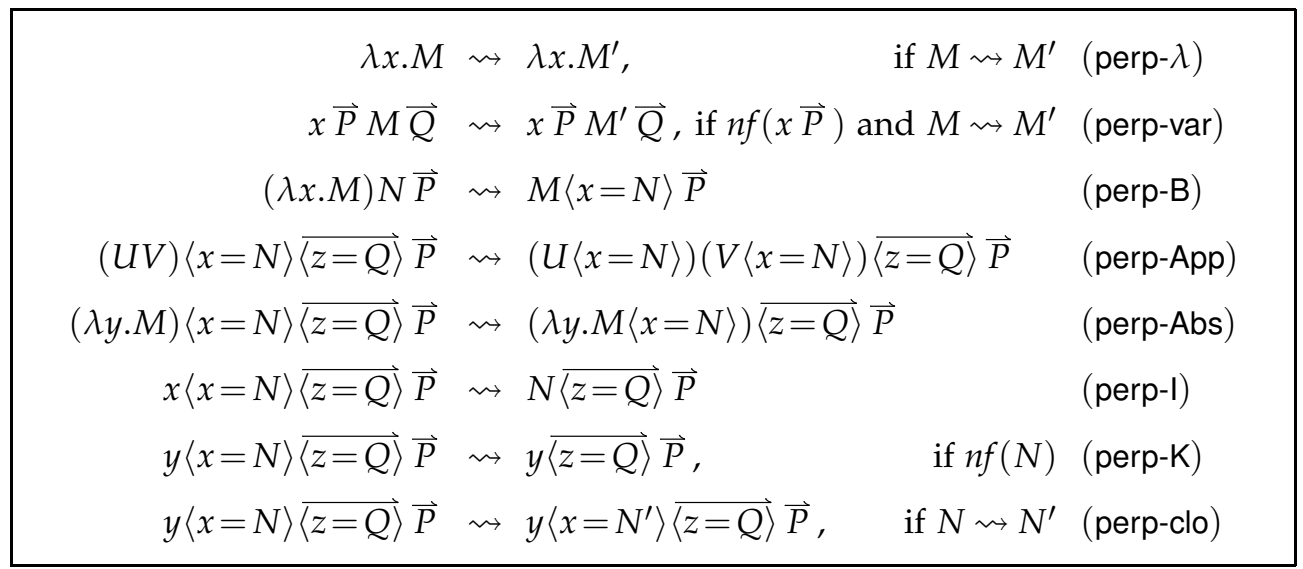

Figure 2: The perpetual strategy and the head-form taxonomy

Theorem 3.7 The following are equivalent

- $M \in \mathcal{S N}$.

- The perpetual strategy terminates on M.

Proof: For the non-trivial direction, examine the inductive characterization of $\mathcal{S N}$ and observe that when $M$ is not strongly normalizing and has the form of the conclusion of one of the inference rules there, one of the hypotheses of the rule is obtained from $M$ by the perpetual strategy.

\section{The system $\mathcal{E}$ of intersection types}

We will consider intersection types as first defined in [15] with a pre-order which takes the idempotence, commutativity and associativity of the intersection type constructor into account.

Definition 4.1 The set of types, ranged over by $\sigma, \tau, \rho, \ldots$, is inductively defined as follows.

$$
\tau_{1}, \tau_{2}::=\varphi\left|\tau_{1} \cap \tau_{2}\right| \tau_{1} \rightarrow \tau_{2}
$$

where $\varphi$ ranges over a denumerable set of type atoms.

The standard pre-ordering $\leq$ on types is the smallest transitive and reflexive relation such that

$$
\begin{array}{r}
\tau_{1} \cap \tau_{2} \leq \tau_{1}, \\
\tau_{1} \cap \tau_{2} \leq \tau_{2}, \\
\text { if } \sigma \leq \tau_{1} \text { and } \sigma \leq \tau_{2} \text { then } \sigma \leq \tau_{1} \cap \tau_{2}
\end{array}
$$

The pre-order defines the equivalence relation on types :

$$
\tau \sim \sigma \text { if and only if } \tau \leq \sigma \text { and } \sigma \leq \tau
$$

In the concrete syntax of types we give, as usual, $\cap$ precedence over $\rightarrow$, right-most outermost brackets will be omitted, and, since the type constructor $\cap$ is associative and commutative, we will write $\sigma \cap \tau \cap \rho$ rather than $(\sigma \cap \tau) \cap \rho$.

The notion of environment is standard, but defining the union of environments requires some care in the presence of the intersection type constructor. 
Definition 4.2 An environment is a partial assignment from variables to types, where each individual assignment is written $(x: \tau)$. Environments are partially ordered as follows.

$$
\Gamma \leq \Gamma^{\prime} \quad \text { iff } \quad\left(x: \tau^{\prime}\right) \in \Gamma^{\prime} \text { implies }(\exists \tau) \cdot(x: \tau) \in \Gamma \text { and } \tau \leq \tau^{\prime}
$$

By abuse of notation, we write $x \in \Gamma$ for $(\exists \tau) .(x: \tau) \in \Gamma . \Gamma \backslash x$ is the environment which does not contain $x$ in its domain and which assigns the same type as $\Gamma$ to the other variables.

Notice that the direction of the ordering $\leq$ on environments may seem at first somewhat counter-intuitive: for example, in the case where for each $\tau$ and $\tau^{\prime}$ we have $\tau=\tau^{\prime}, \Gamma \leq$ $\Gamma^{\prime}$ means $\Gamma \supseteq \Gamma^{\prime}$. But as we will see, $\Gamma \leq \Gamma^{\prime}$ can be thought of as an extension of $\leq$ to environments.

$$
\text { Definition } 4.3 \begin{aligned}
\Gamma_{1} \sqcap \Gamma_{2}= & \left\{(x: \tau) \mid(x: \tau) \in \Gamma_{1} \& x \notin \Gamma_{2}\right\} \cup \\
& \left\{(x: \tau) \mid(x: \tau) \in \Gamma_{2} \& x \notin \Gamma_{1}\right\} \cup \\
& \left\{\left(x: \tau_{1} \cap \tau_{2}\right) \mid\left(x: \tau_{1}\right) \in \Gamma_{1} \&\left(x: \tau_{2}\right) \in \Gamma_{2}\right\} \\
\Gamma,(x: \tau)= & \Gamma \backslash x \cup\{(x: \tau)\}
\end{aligned}
$$

For example, $\left\{\left(x: \tau_{1}\right)\right\} \sqcap\left\{\left(x: \tau_{2}\right)\right\}$ denotes $\left\{\left(x: \tau_{1} \cap \tau_{2}\right)\right\}$, while $\left\{\left(x: \tau_{1}\right)\right\},\left(x: \tau_{2}\right)$ denotes $\left\{\left(x: \tau_{2}\right)\right\}$.

Lemma $4.4 \bullet \Gamma_{1} \sqcap \Gamma_{2} \leq \Gamma_{1}$ and $\Gamma_{1} \sqcap \Gamma_{2} \leq \Gamma_{2}$.

- If $\Gamma_{1} \leq \Gamma$ and $\Gamma_{2} \leq \Gamma$ then $\Gamma_{1} \sqcap \Gamma_{2} \leq \Gamma$.

Proof: These are routine verifications.

As discussed in the introduction, the key of our type assignment are non-standard cut-rules. These will appear in the definition below as (drop) and (K-cut).

Definition 4.5 (Type Assignment Rules) The system $\mathcal{E}$ of type assignment for terms in $\lambda \mathrm{x}$ is defined as follows:

$$
\begin{array}{rlr}
\text { (start) } & \frac{\Gamma \vdash x: \sigma}{\Gamma \vdash}((x: \sigma) \in \Gamma) & (\rightarrow \mathrm{I}) \frac{\Gamma,(x: \sigma) \vdash M: \tau}{\Gamma \vdash \lambda x: M: \sigma \rightarrow \tau} \\
\text { (cut) } \frac{\Gamma,(x: \sigma) \vdash M: \tau \quad \Gamma \vdash N: \sigma}{\Gamma \vdash M\langle x=N\rangle: \tau} & (\rightarrow \mathrm{E}) \frac{\Gamma \vdash M: \sigma \rightarrow \tau \quad \Gamma \vdash N: \sigma}{\Gamma \vdash M N: \tau} \\
\text { (drop) } \frac{\Gamma \vdash M: \tau \quad \Delta \vdash N: \sigma}{\Gamma \vdash M\langle x=N\rangle: \tau}(x \notin a v(M)) & (\cap \mathrm{I}) \frac{\Gamma \vdash M: \sigma \quad \Gamma \vdash M: \tau}{\Gamma \vdash M: \sigma \cap \tau} \\
\text { (K-cut) } \frac{\Gamma \vdash M: \tau \quad \Delta \vdash N: \sigma}{\Gamma \vdash M\langle x=N\rangle: \tau}(x \notin \Gamma) & (\cap \mathrm{E}) \frac{\Gamma \vdash M: \sigma_{1} \cap \sigma_{2}}{\Gamma \vdash M: \sigma_{i}}(i \in\{1,2\})
\end{array}
$$

We write $\Gamma \vdash M: \sigma$ if there exists a derivation constructed using the above rules that has this statement as its conclusion.

Example 4.6 Figure 3 shows a typing derivation for the term $z\langle y=x x\rangle\langle x=\lambda a . a a\rangle$ of Example 1.1. 


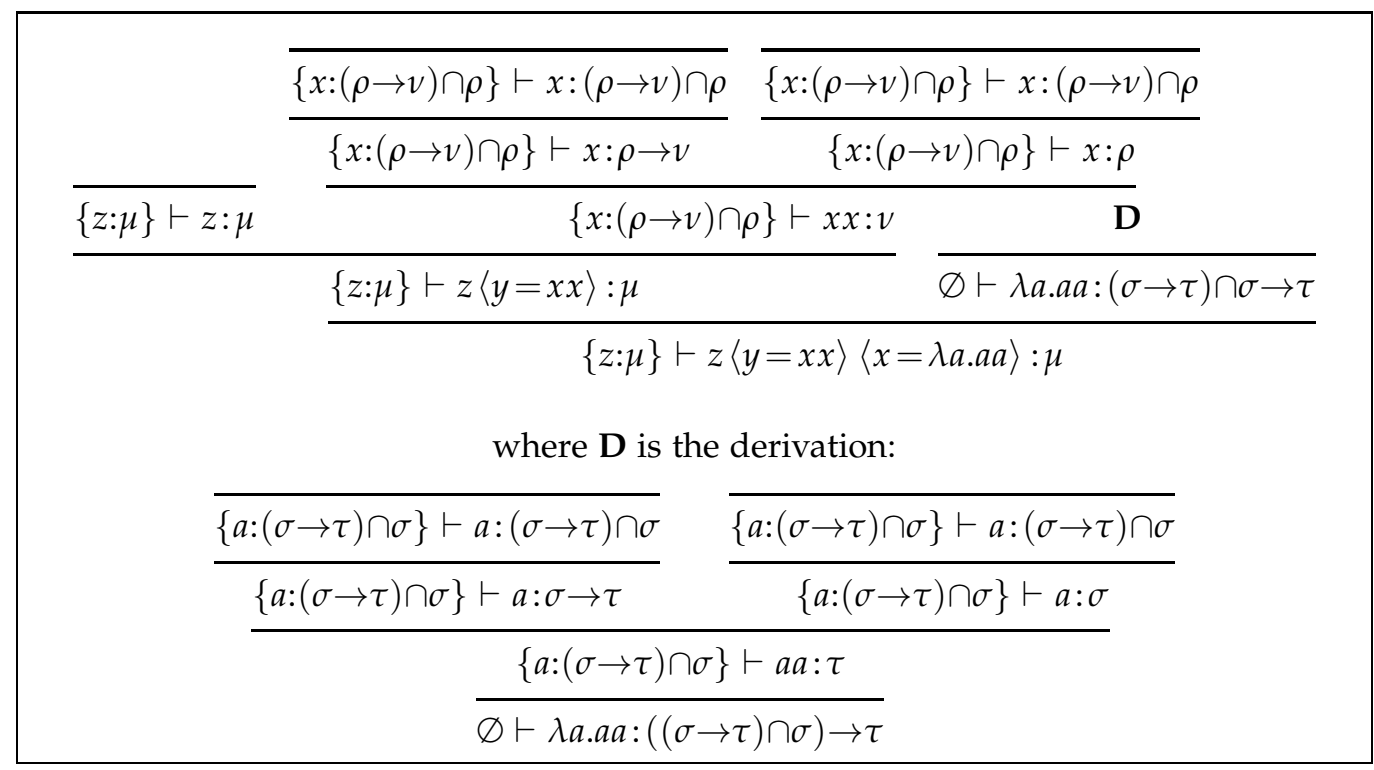

Figure 3: A typing derivation

The type system of [20] is obtained by removing the inference rules (drop) and (K-cut): the point of view taken there was that a closure $M\langle x=N\rangle$ should always have the same typing behaviour as the B-redex $(\lambda x . M) N$ which yields it. This is a plausible strategy since B-reduction involves no (immediate) erasing of sub-terms, even when $x$ is not free in $M$; and indeed the resulting system - in the presence of a universal type - yields the expected characterizations of head-normalizing and left-most-normalizing terms. But as we have seen in Example 1.1, this system failed to provide a characterization of the strongly normalizing terms. This example makes clear that we must allow the type system to distinguish between certain B-redexes and their contractions.

One might note that, in Example 1.1, the input variable of the B-redex in $M_{1}$ does not occur free in the function body (i.e., we have a "K-redex" in LC). This suggests modifying the cut-rule to obtain one which, when typing $M\langle x=N\rangle$ with $x$ not free in $M$, relaxes the typing hypothesis for $N$ to merely ask that it be typable under some environment. This seems particularly appropriate since it echoes the hypotheses of the Subject Expansion Theorem in treatments of intersection types for LC. But such a rule doesn't work: it is still too restrictive. For example, the reader can easily check that the term $z\langle y=x x\rangle\langle x=\lambda a . a a\rangle$ cannot be typed in such a system since $x \in f_{v}(z\langle y=x x\rangle)$, but it is clearly strongly normalizing. This example should motivate our notion of available variable occurrence and the corresponding typing rule (drop).

One can also observe that no premise for $x$ is necessary when typing $z$ in $z\langle y=x x\rangle\langle x=\lambda a . a a\rangle$ and this leads to the introduction of rule (K-cut).

Figure 3 shows how the term $z\langle y=x x\rangle\langle x=\lambda a . a a\rangle$ can be typed in system $\mathcal{E}$.

Notice that rule (cut) has no side-condition, and therefore, when $x \notin a v(M)$ and $\Gamma \vdash N: \sigma$, one can freely use (cut) or (drop); when $x \notin \Gamma$ and $\Gamma \vdash N: \sigma$, one can freely use (cut) or (K-cut).

We now state some elementary properties of the type system, which highlight the relations between the non-standard cut rules.

Lemma 4.7 i) If $\Gamma^{\prime} \leq \Gamma, \tau \leq \tau^{\prime}$ and $\Gamma \vdash M: \tau$ then $\Gamma^{\prime} \vdash M: \tau^{\prime}$.

ii) If $x \in a v(M)$, then $\Gamma \vdash M: \tau$ implies $x \in \Gamma$.

iii) If $x \notin a v(M)$, then $\Gamma \vdash M: \tau$ implies $\Gamma \backslash x \vdash M: \tau$. 
iv) If $x \notin a v(M)$, then $\Gamma \vdash M: \tau$ implies $\Gamma,(x: \sigma) \vdash M: \tau$ for any type $\sigma$.

Proof: By induction on the structure of derivations, with the exception of part (iv) which follows immediately from parts (i) and (iii).

- Before proving part (i) it is useful to make the following observation. Let $M_{z}^{y}$ denote the result of substituting (in the traditional sense) $y$ for $z$ in $M$, and let $\Gamma_{z}^{y}$ be the obvious extension of this notion to environments. If $\Gamma \vdash M: \tau$, then $\Gamma_{z}^{y} \vdash M_{z}^{y}: \tau$ (this follows by a straightforward induction). Now, in proving part (i), the only non-trivial case is when the last applied rule is ( $\mathrm{K}$-cut):

$$
\text { (K-cut) }: \frac{\Gamma \vdash P: \tau \quad \Delta \vdash N: \sigma}{\Gamma \vdash P\langle y=N\rangle: \tau}(y \notin \Gamma)
$$

Now, if $y$ did not occur in $\Gamma^{\prime}$, the argument would be a simple appeal to the induction hypothesis. But there is no reason to assume this, so we have to work a little. Let $y^{\prime}$ be a fresh variable, not occurring (free) in $\Gamma^{\prime}, \Delta, P$, or $N$. Since $\Gamma^{\prime} \leq \Gamma$, we know that $y^{\prime}$ does not occur in $\Gamma$. By our observation about the preservation of derivations under ordinary substitution, $\Gamma \vdash P_{y}^{y^{\prime}}: \tau$. So by induction $\Gamma^{\prime} \vdash P_{y}^{y^{\prime}}: \tau^{\prime}$. Thus $\Gamma^{\prime} \vdash P_{y}^{y^{\prime}}\left\langle y^{\prime}=N\right\rangle: \tau^{\prime}$ by (K-cut). But $P_{y}^{y^{\prime}}\left\langle y^{\prime}=N\right\rangle$ is $\alpha$-equivalent with $P\langle y=N\rangle$, so we are done.

- For part (ii), three cases have to be looked at. The first one is when $M$ is $P\langle y=N\rangle$ and the derivation ends with

$$
\text { (cut) }: \frac{\Gamma,(y: \sigma) \vdash P: \tau \quad \Gamma \vdash N: \sigma}{\Gamma \vdash P\langle y=N\rangle: \tau}
$$

Since $x \in a v(M)$, by Lemma 2.3, $x$ is free in $M$ and by the variable convention and the fact that $y$ is bound, we get $x \neq y$. By the definition of available variable, $x$ available in $M \equiv P\langle y=N\rangle$ means that $x \in a v(P)$ or $x \in a v(N)$. In both cases the induction hypothesis yields $x \in \Gamma$. The other cases are

$$
\begin{aligned}
(\text { drop }): & \frac{\Gamma \vdash P: \tau \quad \Delta \vdash N: \sigma}{\Gamma \vdash P\langle y=N\rangle: \tau}(y \notin a v(P)) \\
\text { (K-cut) }: & \frac{\Gamma \vdash P: \tau \quad \Delta \vdash N: \sigma}{\Gamma \vdash P\langle y=N\rangle: \tau}(y \notin \Gamma)
\end{aligned}
$$

In the case of (K-cut), notice that, by induction, $y \notin \Gamma$ implies $y \notin a v(P)$. So in each case, from $x \in a v(M)$ we get $x \in a v(P)$. We may then conclude, by induction, that $x \in \Gamma$.

\subsection{Derivable rules}

By Definition 4.5, the rules of system $\mathcal{E}$ are (start), $(\rightarrow \mathrm{I}),(\rightarrow \mathrm{E}),(\cap \mathrm{I}),(\cap \mathrm{E}),($ cut), (drop), and (K-cut). $\mathcal{D} \mathcal{L} \mathcal{L}$ is the system obtained from $\mathcal{E}$ by dropping rule (K-cut) and $v \mathcal{B D}$ is the systems obtained from $\mathcal{E}$ by dropping rule (drop). We will write $\Gamma \vdash_{\mathcal{D} \mathcal{L} \mathcal{L}} M: \sigma$ if there exists a derivation with rules in $\mathcal{D} \mathcal{L} \mathcal{L}$ that has this as its conclusion, and similarly $\Gamma \vdash_{v \mathcal{B D}} M: \sigma$.

We will show that these systems have the same typing power as system $\mathcal{E}$, so we can say that just one of the rules (K-cut) and (drop) suffices.

Lemma 4.8 i) Rule (K-cut) is derivable in system $\mathcal{D} \mathcal{L} \mathcal{L}$.

ii) Rule (drop) is derivable in system $v \mathcal{B D}$. 
Proof: i) Each application of rule (K-cut)

$$
\text { (K-cut) }: \frac{\Gamma \vdash P: \tau \quad \Delta \vdash N: \sigma}{\Gamma \vdash P\langle y=N\rangle: \tau}(y \notin \Gamma)
$$

can be replaced by an application of rule (drop), since, by Lemma 4.7(ii), $y \notin \Gamma$ implies $y \notin a v(P)$.

ii) Consider an application of rule (drop):

$$
\text { (drop) : } \frac{\Gamma \vdash P: \tau \quad \Delta \vdash N: \sigma}{\Gamma \vdash P\langle y=N\rangle: \tau}(y \notin a v(P))
$$

By Lemma 4.7(iii), $\Gamma \backslash y \vdash P: \tau$. Then the (K-cut) rule yields $\Gamma \backslash y \vdash P\langle y=N\rangle: \tau$. Then, by Lemma 4.7(iv), we have $\Gamma \vdash P\langle y=N\rangle: \tau$.

From the above Lemma we easily get:

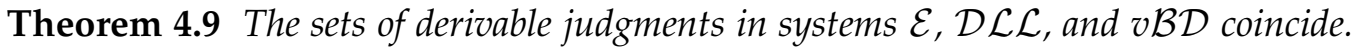

\section{Typing strongly normalizing terms}

As usual for type assignment systems, we have a Generation Lemma. We will use a generic notation for intersection types, $\sigma_{1} \cap \ldots \cap \sigma_{n}$, and assume that then each $\sigma_{i}$ is not an intersection type.

Lemma 5.1 (Generation Lemma) i) $\Gamma \vdash x: \sigma$ if and only if there exists $(x: \tau) \in \Gamma$ such that $\tau \leq \sigma$.

ii) $\Gamma \vdash M N: \sigma$ if and only if there exist $n$, and $\sigma_{i}, \tau_{i}(i \in \underline{n})$ such that $\sigma \sim \sigma_{1} \cap \ldots \cap \sigma_{n}$, and

$\Gamma \vdash M: \tau_{i} \rightarrow \sigma_{i}$ and $\Gamma \vdash N: \tau_{i}$.

iii) $\Gamma \vdash \lambda x . M: \sigma$ if and only if there exist $n$, and $\rho_{i}, \tau_{i}(i \in \underline{n})$ such that

$\sigma \sim\left(\rho_{1} \rightarrow \tau_{1}\right) \cap \ldots \cap\left(\rho_{n} \rightarrow \tau_{n}\right)$, and $\Gamma,\left(x: \rho_{i}\right) \vdash M: \tau_{i}$ whenever $i \in \underline{n}$.

iv) $\Gamma \vdash M\langle x=N\rangle: \sigma$ if and only if either

a) $x \in$ av $(M)$, and there exists $\tau$ such that $\Gamma,(x: \tau) \vdash M: \sigma$ and $\Gamma \vdash N: \tau$, or

b) $x \notin a v(M), \Gamma \vdash M: \sigma$ and there exist $\Delta, \tau$ such that $\Delta \vdash N: \tau$ (in other words: $N$ is typable (in some environment)).

Proof: The right-to-left implications immediately follow from the typing rules. The converse follows by easy induction on the structure of derivations. For part (iv), notice that Theorem 4.9 allows us to skip the (K-cut) rule. If the last applied rule is $(\cap \mathrm{I})$ we can use Lemma 4.7(i) and rule $(\cap \mathrm{I})$.

A minimal requirement of our system is that it satisfies the subject reduction property (SR). We will show SR for the reduction $\lambda \mathrm{x}_{\mathrm{gc}}$ : this gives us SR for $\lambda \mathrm{x}$ for free.

Theorem 5.2 (Subject Reduction) If $M \longrightarrow N$, then $\Gamma \vdash M: \tau$ implies $\Gamma \vdash N: \tau$.

Proof: By induction on the definition of the reduction relation, ' $\longrightarrow$ '. We only show the base cases.

(B): Then $\Gamma \vdash(\lambda x . M) N: \sigma$, and, by Lemma 5.1(ii), there exist types $\sigma_{i}, \rho_{i}(i \in \underline{n})$ such that $\sigma \sim \sigma_{1} \cap \ldots \cap \sigma_{n}$, and, for all $i \in \underline{n}, \Gamma \vdash \lambda x . M: \rho_{i} \rightarrow \sigma_{i}$ and $\Gamma \vdash N: \rho_{i}$. Then, by Lemma 5.1(iii) 
$\Gamma,\left(x: \rho_{i}\right) \vdash M: \sigma_{i}$, and therefore, $\Gamma \vdash M\langle x=N\rangle: \sigma_{i}$ by rule (cut). So, by rule $(\cap \mathrm{l})$, $\Gamma \vdash M\langle x=N\rangle: \sigma$.

(App): Then $\Gamma \vdash(M N)\langle x=P\rangle: \sigma$. Let $\sigma \sim\left(\sigma_{1} \cap \ldots \cap \sigma_{n}\right)$, then, by Lemma 5.1(iv), we have two cases:

$x \in a v(M N) \&(\exists \tau) . \Gamma,(x: \tau) \vdash M N: \sigma \& \Gamma \vdash P: \tau$ : Then we have $x \in a v(M)$ or $x \in a v(N)$, and, by Lemma 5.1(ii), for every $i \in \underline{n}$, there exists $\rho_{i}$ such that $\Gamma,(x: \tau) \vdash M: \rho_{i} \rightarrow \sigma_{i}$ and $\Gamma,(x: \tau) \vdash N: \rho_{i}$. Then $\Gamma \vdash M\langle x=P\rangle: \rho_{i} \rightarrow \sigma_{i}$ by rule (cut), and $\Gamma \vdash N\langle x=P\rangle: \rho_{i}$.

$x \notin a v(M N), \Gamma \vdash M N: \sigma \&(\exists \Delta, \tau) . \Delta \vdash P: \tau$ : Then we have $x \notin a v(M)$ and $x \notin a v(N)$. As above, by Lemma 5.1(ii), for every $i \in \underline{n}$, there exists $\rho_{i}$ such that $\Gamma \vdash M: \rho_{i} \rightarrow \sigma_{i}$ and $\Gamma \vdash N: \rho_{i}$. Then, by rule (drop), $\Gamma \vdash M\langle x=P\rangle: \rho_{i} \rightarrow \sigma_{i}$ and also $\Gamma \vdash N\langle x=P\rangle: \rho_{i}$.

In both cases, by rule $(\rightarrow \mathrm{E})$, we get $\Gamma \vdash(M\langle x=P\rangle)(N\langle x=P\rangle): \sigma_{i}$, so by rule $(\cap \mathrm{I}), \Gamma \vdash(M\langle x=P\rangle)(N\langle x=P)$ (Abs): Then $\Gamma \vdash(\lambda y \cdot M)\langle x=N\rangle: \sigma$. Let $\sigma \sim\left(\sigma_{1} \cap \ldots \cap \sigma_{n}\right)$. By Lemma 5.1(iv), we have two cases:

$x \in \operatorname{av}(M) \&(\exists \tau) \Gamma,(x: \tau) \vdash \lambda y \cdot M: \sigma \& \Gamma \vdash N: \tau$ : By Lemma 5.1(iii), for $i \in \underline{n}$, there exist $\rho_{i}, \mu_{i}$ such that $\sigma_{i} \sim \rho_{i} \rightarrow \mu_{i}$ and $\Gamma,(x: \tau),\left(y: \rho_{i}\right) \vdash M: \mu_{i}$. Then we get

$\Gamma,\left(y: \rho_{i}\right) \vdash M\langle x=N\rangle: \mu_{i}$ by rule (cut).

$x \notin a v(M), \Gamma \vdash \lambda y \cdot M: \sigma \&(\exists \Delta, \tau) \Delta \vdash N: \tau:$ As above, there exist $\rho_{i}, \mu_{i}$ such that $\sigma_{i} \sim \rho_{i} \rightarrow \mu_{i}$ and $\Gamma \backslash x,\left(y: \rho_{i}\right) \vdash M: \mu_{i}$. Then $\Gamma,\left(y: \rho_{i}\right) \vdash M\langle x=N\rangle: \mu_{i}$ by rule (drop).

In both cases, we obtain $\Gamma \vdash \lambda y .(M\langle x=N\rangle): \sigma_{i}$ by rule $(\rightarrow I)$, and, by rule $(\cap \mathrm{I})$, also $\Gamma \vdash \lambda y \cdot(M\langle x=N\rangle): \sigma$.

(Varl): Then $\Gamma \vdash x\langle x=N\rangle: \sigma$, and, by Lemma 5.1(iv) $\Gamma,(x: \tau) \vdash x: \sigma$ and $\Gamma \vdash N: \tau$ for a certain $\tau$. Then, by Lemma 5.1(i), $\tau \leq \sigma$, and, by Lemma 4.7(i), $\Gamma \vdash N: \sigma$.

(gc): Then $\Gamma \vdash M\langle x=N\rangle: \sigma$ and $x \notin a v(M)$. Then, by Lemma 5.1(iv), $\Gamma \vdash M: \sigma$.

Normal forms in $\lambda \mathrm{x}$ are the same as in LC, and the type system $\mathcal{E}$ is an extension of the standard system of intersection types for LC. Therefore we get the typability of all normal forms for free. Moreover, we show that $\lambda$-free normal forms (that is to say, normal forms which are not $\lambda$-abstractions) have arbitrary types: this also holds in the the standard system of intersection types.

Lemma 5.3 (NORMAL FORMS ARE TYPABLE) Let $M$ be a normal form.

i) If $M$ is $\lambda$-free and $\tau$ is a type, then there is an environment in which $M$ has type $\tau$.

ii) $M$ is typable in some environment.

Proof: By simultaneous structural induction on $M$.

- If $M$ is a variable, both statements hold.

- If $M \equiv x M_{1} \ldots M_{n}$, where $M_{1}, \ldots, M_{n}$ are normal forms, then by induction there are, for $i \in \underline{n}, \Gamma_{i}, \tau_{i}$ such that $\Gamma_{i} \vdash M_{i}: \tau_{i}$. Then $\Gamma_{1} \sqcap \ldots \Gamma_{n} \sqcap\left\{x: \tau_{1} \rightarrow \ldots \rightarrow \tau_{n} \rightarrow \tau\right\} \vdash M: \tau$. So $M$ is typable with an arbitrary type $\tau$ in a suitable environment.

- If $M \equiv \lambda x \cdot M^{\prime}$, then by induction (second statement), there are $\Gamma$ and $\tau$ such that $\Gamma \vdash M^{\prime}: \tau$. Then $\Gamma,(x: \sigma) \vdash M^{\prime}: \tau$, where either $(x: \sigma) \in \Gamma$ or $x \notin \Gamma$ and $\sigma$ is any type. Hence, $\Gamma \backslash x \vdash M: \sigma \rightarrow \tau$.

The key property to obtain the typability of all strongly normalizing terms is the preservation of typability when we expand using the perpetual strategy. This comes as a corollary of the following more technical theorem. 
Theorem 5.4 (Subject Expansion) If $M \rightsquigarrow N$ in one step, then

i) if the rule applied in the reduction is not (B): $\Gamma \vdash N: \tau \Rightarrow \Gamma \vdash M: \tau$

ii) if the rule applied in the reduction is $(\mathrm{B})$ :

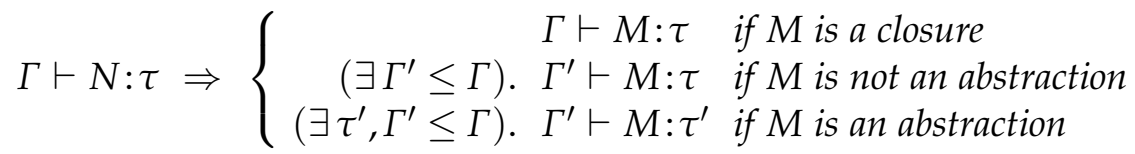

Proof: i) By induction on the structure $M$. The base case is when $M$ is its own perpetual redex: let us reason by cases on the rule used.

(App): We assume $\Gamma \vdash P\langle x=U\rangle Q\langle x=U\rangle: \sigma$, and want to prove $\Gamma \vdash(P Q)\langle x=U\rangle: \sigma$. By Lemma 5.1(ii), there are types $\tau_{i}, \sigma_{i}(i \in \underline{n})$ such that $\sigma \sim\left(\sigma_{1} \cap \ldots \cap \sigma_{n}\right)$, and

$$
(\forall i \in \underline{n}) . \Gamma \vdash P\langle x=U\rangle: \tau_{i} \rightarrow \sigma_{i} \& \Gamma \vdash Q\langle x=U\rangle: \tau_{i}
$$

By rule $(\cap \mathrm{l})$ it suffices to prove that $(\forall i \in \underline{n}) . \Gamma \vdash(P Q)\langle x=U\rangle: \sigma_{i}$. If $x \notin a v(P)$ and $x \notin$ av $(Q)$, we apply Lemma 5.1(iv), which gives $\Gamma \vdash P: \tau_{i} \rightarrow \sigma_{i}$ and $\Gamma \vdash Q: \tau_{i}$, as well as that $U$ is typable. Consequently, $\Gamma \vdash P Q: \sigma_{i}$ and finally, by rule (drop), $\Gamma \vdash(P Q)\langle x=U\rangle: \sigma_{i}$. If $x \in a v(P)$ or $x \in a v(Q)$, it suffices to prove

$$
\left(\exists \tau_{i}^{\prime}\right) . \Gamma \vdash U: \tau_{i}^{\prime} \quad \& \quad \Gamma,\left(x: \tau_{i}^{\prime}\right) \vdash P: \tau_{i} \rightarrow \sigma_{i} \quad \& \quad \Gamma,\left(x: \tau_{i}^{\prime}\right) \vdash Q: \tau_{i}
$$

(which induces by rule (cut), $\Gamma \vdash(P Q)\langle x=U\rangle: \sigma_{i}$ ). In each case, we apply Lemma 5.1(iv) on both $P$ and $Q$.

* If $x \in a v(P)$ and $x \notin a v(Q)$, we get $\mu$ such that $\Gamma,(x: \mu) \vdash P: \tau_{i} \rightarrow \sigma_{i}$ and $\Gamma \vdash U: \mu$. Taking $\tau_{i}^{\prime}$ to be $\mu$, we use 4.7(iv) on $Q$ to get the result.

* If $x \notin a v(P)$ and $x \in a v(Q)$, we get $v$ such that $\Gamma,(x: v) \vdash Q: \tau_{i}$ and $\Gamma \vdash U: v$. Taking $\tau_{i}^{\prime}$ to be $v$, we use 4.7(iv) on $P$ to get the result.

* If $x \in a v(P)$ and $x \in a v(Q)$, we get $\mu$ and $v$, such that

$$
\Gamma \vdash U: \mu, \quad \& \quad \Gamma \vdash U: v, \quad \& \quad \Gamma,(x: \mu) \vdash P: \tau_{i} \rightarrow \sigma_{i} \quad \& \quad \Gamma,(x: v) \vdash Q: \tau_{i}
$$

If we set $\tau_{i}^{\prime}$ to $\mu \cap \nu$ we get the result.

(Abs): Suppose $M \equiv(\lambda y \cdot P)\langle x=U\rangle$ and $N \equiv \lambda y .(P\langle x=U\rangle)$. By Barendregt's convention, $y \notin a v(U)$ and $x \neq y$; then $x \in a v(P)$ if and only if $x \in a v(\lambda y . P)$. We assume $\Gamma \vdash \lambda y .(P\langle x=U\rangle): \sigma$, and want to prove $\Gamma \vdash(\lambda y . P)\langle x=U\rangle: \sigma$. Using Lemma 5.1(iii), we have types $\tau_{i}, \sigma_{i}(i \in \underline{n})$ such that $\sigma \sim\left(\tau_{1} \rightarrow \sigma_{1}\right) \cap \ldots \cap\left(\tau_{n} \rightarrow \sigma_{n}\right)$ and $(\forall i \in \underline{n}) . \Gamma,\left(y: \tau_{i}\right) \vdash P\langle x=U\rangle: \sigma_{i}$. By rule $(\cap \mathrm{l})$ it suffices to prove that $(\forall i \in \underline{n}) . \Gamma \vdash(\lambda y . P)\langle x=U\rangle: \tau_{i} \rightarrow \sigma_{i}$. We apply Lemma 5.1(iv) on $\Gamma,\left(y: \tau_{i}\right) \vdash P\langle x=U\rangle: \sigma_{i}$ and thereby,

* If $x \in a v(P)$ we get $\mu$ such that $\Gamma,\left(y: \tau_{i}\right),(x: \mu) \vdash P: \sigma_{i}$ and $\Gamma,\left(y: \tau_{i}\right) \vdash U: \mu$. Since $y \notin a v(U)$, applying Lemma 4.7(i) we get $\Gamma,(x: \mu),\left(y: \tau_{i}\right) \vdash P: \sigma_{i}$ and $\Gamma \vdash U: \mu$.

* If $x \notin a v(P)$ we get that $U$ is typable and $\Gamma,\left(y: \tau_{i}\right) \vdash P: \sigma_{i}$.

In both cases, we get the required result by applying first rule $(\rightarrow I)$ and then respectively rules (cut) or (drop).

(Varl): If $\Gamma \vdash U: \tau$, then clearly $\Gamma,(x: \tau) \vdash x: \tau$ and $\Gamma \vdash x\langle x=U\rangle: \tau$.

(Vark): Then $U$ is a normal form, and, by Lemma 5.3, $U$ is typable. We assume $\Gamma \vdash y: \sigma$, and rule (drop) yields $\Gamma \vdash y\langle x=U\rangle: \sigma$. 
Now for the induction step, since the environment and the type of $M$ are the same as of $N$, the proof is easy using the same typing tree.

ii) Again, the proof is by induction on the structure of $M$.

$M$ is its own perpetual redex: We wish to prove: if $\Gamma \vdash P\langle x=U\rangle: \tau$, then

$\left(\exists \Gamma^{\prime \prime} \leq \Gamma\right) . \Gamma^{\prime \prime} \vdash(\lambda x . P) U: \tau$.

* If $x \in a v(P)$, we have $\left(\exists \tau^{\prime}\right) . \Gamma,\left(x: \tau^{\prime}\right) \vdash P: \tau \& \Gamma \vdash U: \tau^{\prime}$, so $\left(\exists \tau^{\prime}\right) . \Gamma \vdash \lambda x . P: \tau^{\prime} \rightarrow \tau \& \Gamma \vdash U: \tau^{\prime}$ which entails $\Gamma \vdash(\lambda x . P) U: \tau$ by rule $(\rightarrow \mathrm{E})$.

* If $x \notin a v(P)$, then, using Lemma 5.1(iv), we have $\Gamma \vdash P: \tau$ and $\left(\exists \Gamma^{\prime}, \tau^{\prime}\right) \cdot \Gamma^{\prime} \vdash U: \tau^{\prime}$. From Lemma 4.7(i), we get $\Gamma,\left(x: \tau^{\prime}\right) \vdash P: \tau$ which yields $\Gamma \vdash \lambda x \cdot P: \tau^{\prime} \rightarrow \tau$ by rule $(\rightarrow \mathrm{I})$. Hence $\left(\exists \Gamma^{\prime}, \tau^{\prime}\right) . \Gamma \vdash \lambda x . P: \tau^{\prime} \rightarrow \tau \& \Gamma^{\prime} \vdash U: \tau^{\prime}$. If we set $\Gamma^{\prime \prime}$ to be $\Gamma \sqcap \Gamma^{\prime} \leq \Gamma$ we get $\Gamma^{\prime \prime} \vdash \lambda x . P: \tau^{\prime} \rightarrow \tau$ and $\Gamma^{\prime \prime} \vdash U: \tau^{\prime}$ which entails $\Gamma^{\prime \prime} \vdash(\lambda x . P) U: \tau$.

$M \equiv \lambda x \cdot M^{\prime}$ : Then $N \equiv \lambda x \cdot N^{\prime}$, where $M^{\prime} \rightsquigarrow N^{\prime}$. We assume $\Gamma \vdash \lambda x \cdot N^{\prime}: \sigma$ and want to prove $\Gamma^{\prime} \vdash \lambda x \cdot M^{\prime}: \sigma^{\prime}$ for some environment $\Gamma^{\prime} \leq \Gamma$ and type $\sigma^{\prime}$. Using Lemma 5.1(iii), we have types $\tau_{i}, \sigma_{i}(i \in \underline{n})$ such that $(\forall i \in \underline{n}) \cdot \Gamma,\left(y: \tau_{i}\right) \vdash N^{\prime}: \sigma_{i}$. Then, by induction, we get $\Gamma^{\prime} \leq \Gamma, \tau_{1}^{\prime}$, and $\sigma_{1}^{\prime}$ such that $\Gamma^{\prime},\left(x: \tau_{1}^{\prime}\right) \vdash M^{\prime}: \sigma_{1}^{\prime}$. Taking $\sigma^{\prime}:=\tau_{1}^{\prime} \rightarrow \sigma_{1}^{\prime}$ we get $\Gamma^{\prime} \vdash \lambda x \cdot M^{\prime}: \sigma^{\prime}$ as required.

$M \equiv M_{1} M_{2}$ where $M$ is not its own perpetual redex: Then $N \equiv N_{1} N_{2}$ where either $M_{1} \rightsquigarrow N_{1}$ or $M_{1}$ is a $\lambda$-free normal form and $M_{2} \rightsquigarrow N_{2}$ (see Definition 3.5). We assume $\Gamma \vdash N_{1} N_{2}: \sigma$, and want to prove $\Gamma^{\prime} \vdash M_{1} M_{2}: \sigma$ for some environment $\Gamma^{\prime} \leq \Gamma$. Using Lemma 5.1(ii), we have types $\tau_{i}, \sigma_{i}(i \in \underline{n})$ such that $\sigma \sim \sigma_{1} \cap \ldots \cap \sigma_{n}$ and

$(\forall i \in \underline{n}) . \Gamma \vdash N_{1}: \tau_{i} \rightarrow \sigma_{i} \& \Gamma \vdash N_{2}: \tau_{i}$. Using Lemma 4.7(i) it suffices to prove that $(\forall i \in \underline{n}) \cdot \Gamma_{i} \vdash M_{1} M_{2}: \sigma_{i}$ for some $\Gamma_{i} \leq \Gamma$ (since then we can take $\Gamma^{\prime}$ to be $\left(\Gamma_{1} \sqcap \ldots \sqcap \Gamma_{n}\right) \leq \Gamma_{i} \leq \Gamma$ ). Now by Definition 3.5, $M_{1}$ cannot be an abstraction, otherwise $M$ would be its own perpetual redex.

* If $M_{1} \rightsquigarrow N_{1}$ and $M_{2} \equiv N_{2}$, then we apply the induction hypothesis to $M_{1}$. Hence we have $\Gamma_{i} \leq \Gamma$ such that $\Gamma_{i} \vdash M_{1}: \tau_{i} \rightarrow \sigma_{i}$, and using Lemma 4.7(i) we get $\Gamma_{i} \vdash M_{2}: \tau_{i}$. Hence $\Gamma_{i} \vdash M_{1} M_{2}: \sigma_{i}$.

* If $M_{2} \rightsquigarrow N_{2}$ and $M_{1} \equiv N_{1}$, then we apply the induction hypothesis to $M_{2}$. Hence we have $\Gamma_{i}^{\prime} \leq \Gamma$ and $\tau_{i}^{\prime}$ such that $\Gamma_{i}^{\prime} \vdash M_{2}: \tau_{i}^{\prime}$. By Definition 3.5 we know that $M_{1}$ is a $\lambda$-free normal form, so applying Lemma 5.3(i) provides an environment $\Gamma^{\prime \prime}$ in which $M_{1}$ has type $\tau_{i}^{\prime} \rightarrow \sigma_{i}$. Now, taking $\Gamma_{i}$ to be $\Gamma_{i}^{\prime} \sqcap \Gamma^{\prime \prime}$, we get $\Gamma_{i} \vdash M_{1} M_{2}: \sigma_{i}$ as required.

$M \equiv M_{1}\left\langle x=M_{2}\right\rangle:$ By Definition 3.5, either:

* The perpetual redex of $M$ is in $M_{2}$, and $M_{1} \equiv y \neq x$ (hence, $N \equiv y\left\langle x=N_{2}\right\rangle$ where $\left.M_{2} \rightsquigarrow N_{2}\right)$. Assume $\Gamma \vdash y\left\langle x=N_{2}\right\rangle: \sigma$. Using Lemma 5.1(iv), we get $\Gamma \vdash y: \sigma$. Now by induction $M_{2}$ is typable. Hence applying rule (drop) we get $\Gamma \vdash y\left\langle x=M_{2}\right\rangle: \sigma$ as required.

* The perpetual redex of $M$ is in $M_{1}$, and $M_{1}$ is a closure (hence, $N \equiv N_{1}\left\langle x=M_{2}\right\rangle$ where $\left.M_{1} \rightsquigarrow N_{1}\right)$. We assume $\Gamma \vdash N_{1}\left\langle x=M_{2}\right\rangle: \sigma$, and want to prove $\Gamma \vdash M_{1}\left\langle x=M_{2}\right\rangle: \sigma$.

$x \in a v\left(N_{1}\right)$ : Then, using Lemma 5.1(iv), we have a type $\tau$ such that $\Gamma,(x: \tau) \vdash N_{1}: \sigma$ and $\Gamma \vdash M_{2}: \tau$. Now we can apply the induction hypothesis to $M_{1}$, which is a closure. We get $\Gamma,(x: \tau) \vdash M_{1}: \sigma$, and then we can apply rule (cut) to get $\Gamma \vdash M_{1}\left\langle x=M_{2}\right\rangle: \sigma$.

$x \notin a v\left(N_{1}\right)$ : Then using Lemma 4.7(iii) we get $\Gamma \backslash x \vdash N_{1}\left\langle x=M_{2}\right\rangle: \sigma$. Then we can 
apply Lemma 5.1(iv), and we have $\Gamma \backslash x \vdash N_{1}: \sigma$ and $M_{2}$ is typable. Now we can apply the induction hypothesis to $M_{1}$, which is a closure. We get $\Gamma \backslash x \vdash M_{1}: \sigma$. Note that since $x \notin(\Gamma \backslash x)$, we can apply rule (K-cut) and get $\Gamma \vdash M_{1}\left\langle x=M_{2}\right\rangle: \sigma$.

Corollary 5.5 (Weak Subject Expansion) If $M \rightsquigarrow N$, then $N$ is typable implies $M$ is typable.

Theorem 5.6 All strongly normalizing terms are typable.

Proof: By induction on the length of the perpetual derivation. For the base case we observe that normal forms are typable (Lemma 5.3(ii)), the induction step follows by Corollary 5.5.

\section{All Typable Terms are Strongly Normalizable}

The general idea of the reducibility method, is to interpret types by suitable sets (saturated and stable sets for Tait [40] and Krivine [27] and admissible relations for Mitchell [34, 35]) of terms (reducible terms) which satisfy the required property (e.g. strong normalization) and then to develop semantics in order to obtain the soundness of the type assignment. A consequence of soundness, the fact that every term typable by a type in the type system belongs to the interpretations of that type, leads to the fact that terms typable in the type system satisfy the required property, since the type interpretations are built up in that way.

In order to develop the reducibility method we consider the applicative structure whose domain are the terms in $\lambda \mathrm{x}$ and where the application is just the application of terms.

Definition 6.1 (REDucible terms) i) We define the collection of set of terms $\mathcal{R}^{\rho}$ inductively over types by:

$$
\begin{aligned}
\mathcal{R}^{\varphi} & =\mathcal{S N} \\
\mathcal{R}^{\sigma \rightarrow \tau} & =\left\{M \mid \forall N \in \mathcal{R}^{\sigma}\left[M N \in \mathcal{R}^{\tau}\right]\right\} \\
\mathcal{R}^{\sigma \cap \tau} & =\mathcal{R}^{\sigma} \cap \mathcal{R}^{\tau} .
\end{aligned}
$$

ii) We define the set $\mathcal{R}$ of reducible terms by: $\mathcal{R}=\left\{M \mid \exists \rho\left[M \in \mathcal{R}^{\rho}\right]\right\}=\bigcup_{\rho \in \mathcal{T}} \mathcal{R}^{\rho}$.

Notice that, if $M \in \mathcal{R}^{\sigma}$, not necessarily there exists a $\Gamma$ such that $\Gamma \vdash M: \sigma$. For example, if $\varphi, \varphi^{\prime}$ are two different type variables, then $\lambda x . x \in \mathcal{R}^{\varphi \rightarrow \varphi^{\prime}}$, since $(\lambda x . x) M \in \mathcal{S N}$ whenever $M \in \mathcal{S N}$, but we cannot derive $\varnothing \vdash \lambda x . x: \varphi \rightarrow \varphi^{\prime}$. Also, since $\lambda x . x \in \mathcal{S N}, \lambda x . x \in \mathcal{R}^{\varphi}$, but we cannot derive $\varnothing \vdash \lambda x . x: \varphi$.

We now show that reducibility implies strong normalization and that all term-variables are reducible. For the latter, it is convenient to show a generalization: all typable strongly normalisable terms that start with a term variable are reducible.

Lemma 6.2 i) $\mathcal{R} \subseteq \mathcal{S N}$.

ii) $x \vec{N} \in \mathcal{S N} \Rightarrow \forall \rho\left[x \vec{N} \in \mathcal{R}^{\rho}\right]$.

Proof: By simultaneous induction on the structure of types.

i) $(\varphi)$ : By Definition 6.1.

$$
\begin{gathered}
(\sigma \rightarrow \tau): \quad M \in \mathcal{R}^{\sigma \rightarrow \tau} \Rightarrow(I H(i i)) M \in \mathcal{R}^{\sigma \rightarrow \tau} \& x \in \mathcal{R}^{\sigma} \Rightarrow(6.1) \\
M x \in \mathcal{R}^{\tau} \Rightarrow(I H(i)) M x \in \mathcal{S N} \Rightarrow M \in \mathcal{S N} . \\
(\sigma \cap \tau): M \in \mathcal{R}^{\sigma \cap \tau} \Rightarrow(6.1) M \in \mathcal{R}^{\sigma} \& M \in \mathcal{R}^{\tau} \Rightarrow(I H(i)) M \in \mathcal{S N} .
\end{gathered}
$$


ii) $\quad(\varphi): x \vec{N} \in \mathcal{S N} \Rightarrow(6.1) x \vec{N} \in \mathcal{R}^{\varphi}$.

$$
\begin{aligned}
& (\sigma \rightarrow \tau): x \vec{N} \in \mathcal{S N} \quad \Rightarrow(3.2, \text { (gen-var) }) \\
& \forall M \in \mathcal{S N}[x \vec{N} M \in \mathcal{S N}] \Rightarrow(I H(i)) \\
& \forall M \in \mathcal{R}^{\sigma}[x \vec{N} M \in \mathcal{S N}] \quad \Rightarrow(I H(i i)) \\
& \forall M \in \mathcal{R}^{\sigma}\left[x \vec{N} M \in \mathcal{R}^{\tau}\right] \quad \Rightarrow(6.1) x \vec{N} \in \mathcal{R}^{\sigma \rightarrow \tau} \\
& (\sigma \cap \tau): x \vec{N} \in \mathcal{S N} \Rightarrow(\operatorname{IH}(i i)) x \vec{N} \in \mathcal{R}^{\sigma} \& x \vec{N} \in \mathcal{R}^{\tau} \Rightarrow(6.1) x \vec{N} \in \mathcal{R}^{\sigma \cap \tau} \text {. }
\end{aligned}
$$

We now show that all sets $\mathcal{R}^{\rho}$ are closed under the rules (subs), (gen-B), (gen-App), (gen-Abs), (gen-I) and (gen-gc). This result is needed in the proof of Theorem 6.4.

Lemma 6.3 (SATURATION) For all $\rho$, the sets $\mathcal{R}^{\rho}$ are $\mathcal{S N}$-saturated.

Proof: All these closures are shown by induction on the structure of types. For the case of a type-variable, $\mathcal{R}^{\varphi}=\mathcal{S N}$, which is $\mathcal{S N}$-saturated (Theorem 3.4). For the rest of the induction, since the proofs are all very similar, we will not show all in detail, but focus on rule (subs). Then:

$$
\begin{aligned}
(\sigma \rightarrow \tau): & (P \overrightarrow{\langle x=N\rangle}\langle y=Q \overrightarrow{\langle x=N\rangle}\rangle) \vec{M} \in \mathcal{R}^{\sigma \rightarrow \tau} & \Rightarrow(6.1) \\
& \forall R \in \mathcal{R}^{\sigma}\left[\left(P \overrightarrow{\langle x=N\rangle}\langle y=Q\langle x=N\rangle) \vec{M} R \in \mathcal{R}^{\tau}\right]\right. & \Rightarrow(I H) \\
& \forall R \in \mathcal{R}^{\sigma}\left[((P\langle y=Q\rangle) \overrightarrow{\langle x=N\rangle}) \vec{M} R \in \mathcal{R}^{\tau}\right] & \Rightarrow(6.1) \\
& \left((P\langle y=Q\rangle) \overline{\langle x=N\rangle) \vec{M} \in \mathcal{R}^{\sigma \rightarrow \tau} .}\right. &
\end{aligned}
$$

$(\sigma \cap \tau)$ : Immediate by Definition 6.1 and induction.

We shall prove our strong normalization result by showing that every typable term is reducible. For this, we need to prove a stronger property: we will show that if we substitute term-variables by reducible terms in a typable term, then we obtain a reducible term. This gives the soundness of our type interpretation.

Theorem 6.4 (Soundness) Suppose $\left\{\left(x_{1}: \mu_{1}\right), \ldots,\left(x_{n}: \mu_{n}\right)\right\} \vdash M: \sigma$, and, for $i \in \underline{n}, N_{i} \in \mathcal{R}^{\mu_{i}}$, with no $x_{j}$ available in any $N_{i}$. Then $M \overline{\langle x=N\rangle} \in \mathcal{R}^{\sigma}$.

Proof: Note that by the variable convention we may assume that for all $1 \leq i, j \leq n, x_{i} \notin f v\left(N_{j}\right)$. The proof is by induction on the structure of derivations. We will use the $\mathcal{S N}$-saturation of the saturated sets (Lemma 6.3) just mentioning the rule names. Let $\Gamma=\left\{\left(x_{1}: \mu_{1}\right), \ldots,\left(x_{n}: \mu_{n}\right)\right\}$.

(start): Then $M \equiv x_{j}$, and $\mu_{j}=\sigma$, for some $j \in \underline{n}$. Since $N_{j} \in \mathcal{R}^{\mu_{j}}, N_{j} \in \mathcal{R}^{\sigma}$. Then, by rules (gen-I) and (gen-gc), $x_{j} \overrightarrow{\langle x=N\rangle} \in \mathcal{R}^{\sigma}$.

$(\rightarrow \mathrm{I})$ : Then $M \equiv \lambda y \cdot M^{\prime}, \sigma=\rho \rightarrow \tau$, and $\Gamma,(y: \rho) \vdash M^{\prime}: \tau$. Let $N \in \mathcal{R}^{\rho}$, then, by induction, $M^{\prime} \overrightarrow{\langle x=N\rangle}\langle y=N\rangle \in \mathcal{R}^{\tau}$. So, by rule (gen-B), $\left(\lambda y \cdot M^{\prime} \overrightarrow{\langle x=N\rangle}\right) N \in \mathcal{R}^{\tau}$, and, by Definition 6.1, $\lambda y \cdot M^{\prime} \overline{\langle x=N\rangle} \in \mathcal{R}^{\rho \rightarrow \tau}$. We can assume $y \notin f v(N)$, so, by rule (gen-Abs), $\left(\lambda y \cdot M^{\prime}\right) \overrightarrow{\langle x=N\rangle} \in$ $\mathcal{R}^{\rho \rightarrow \tau}$.

$(\rightarrow \mathrm{E})$ : Then $M \equiv M_{1} M_{2}$ and there exists $\tau$ such that $\Gamma \vdash M_{1}: \tau \rightarrow \sigma$ and $\Gamma \vdash M_{2}: \tau$. By induction, $M_{1} \overrightarrow{\langle x=N\rangle} \in \mathcal{R}^{\tau \rightarrow \sigma}$ and $M_{2} \overrightarrow{\langle x=N\rangle} \in \mathcal{R}^{\tau}$. But then, by Definition 6.1, $M_{1} \overrightarrow{\langle x=N\rangle} M_{2} \overrightarrow{\langle x=N\rangle} \in \mathcal{R}^{\sigma}$, so, by rule (gen-App), $\left(M_{1} M_{2}\right) \overrightarrow{\langle x=N\rangle} \in \mathcal{R}^{\sigma}$.

$(\cap \mathrm{I})$ : Then $\sigma \equiv \sigma_{1} \cap \sigma_{2}$ and, for $i \in \underline{2}, \Gamma \vdash M: \sigma_{i}$. So, by induction, $M \overrightarrow{\langle x=N\rangle} \in \mathcal{R}^{\sigma_{1}}$ and $M \overrightarrow{\langle x=N\rangle} \in \mathcal{R}^{\sigma_{2}}$, so, by Definition 6.1, $M \overrightarrow{\langle x=N\rangle} \in \mathcal{R}^{\sigma}$.

$(\cap \mathrm{E})$ : Then there exists $\tau$ such that $\Gamma \vdash M: \sigma \cap \tau$, and, by induction, $M \overrightarrow{\langle x=N\rangle} \in \mathcal{R}^{\sigma \cap \tau}$. Then, by Definition 6.1, $M \overrightarrow{\langle x=N\rangle} \in \mathcal{R}^{\sigma}$.

(cut): Here $M \equiv P\langle y=Q\rangle$, and there exists $\tau$ such that $\Gamma,(y: \tau) \vdash P: \sigma$ and $\Gamma \vdash Q: \tau$. Then, 
by induction on the right-hand hypothesis, $Q \overrightarrow{\langle x=N\rangle} \in \mathcal{R}^{\tau}$. Then again by induction, but now on the left-hand hypothesis, $P \overrightarrow{\langle x=N\rangle}\langle y=Q \overrightarrow{\langle x=N\rangle}\rangle \in \mathcal{R}^{\sigma}$. So, by rule (subs), $(P\langle y=Q\rangle) \overrightarrow{\langle x=N\rangle} \in \mathcal{R}^{\sigma}$.

(drop): Here $M \equiv P\langle y=Q\rangle, \Gamma \vdash P: \sigma, y \notin \Gamma$ and there exist $\Delta, \tau$ such that $\Delta \vdash Q: \tau$. By induction $P \overline{\langle x=N\rangle} \in \mathcal{R}^{\sigma}$. Since $y \notin a v(P)$ we may use closure of $\mathcal{R}^{\sigma}$ under rule (gen-gc) to conclude that $(P\langle y=Q\rangle) \overrightarrow{\langle x=N\rangle} \in \mathcal{R}^{\sigma}$. To be able to apply that rule, we need that $Q \in \mathcal{S N}$; notice that by induction on the derivation for $Q, Q \in \mathcal{R}^{\tau}$, so, by Lemma 6.2(i), $Q \in \mathcal{S N}$.

(K-cut): The proof is very similar to the (drop) case; we may also use to Theorem 4.9.

Theorem 6.5 If $\Gamma \vdash M: \sigma$ for some $\Gamma$, $\sigma$ then $M \in \mathcal{S N}$.

Proof: Suppose $\Gamma$ is $\left\{\left(x_{1}: \rho_{1}\right), \ldots,\left(x_{m}: \rho_{m}\right)\right\}$. By Lemma 6.2(ii), all term-variables are reducible for any type, so, by Theorem 6.4, for all $M, M \overrightarrow{\langle x=y\rangle}$ is reducible, where $\vec{y}$ are fresh. By Lemma $6.2(i)$ the term $M \overrightarrow{\langle x=y\rangle}$ is strongly normalizing, and since $M$ is a subterm, the result follows.

\section{Characterizing weak normalization and head normalization}

The system $\mathcal{E}$ is obtained from the system $\mathcal{D}$ of [20] by adding the rules (drop) and (K-cut). The system $\mathcal{D}_{\omega}$ is the extension of $\mathcal{D}$ obtained by adding a universal type $\omega$ : this type was first added to intersection type assignment in [39]. The main feature of systems with intersection and $\omega$ is that typing is invariant under any conversion of subjects. In [20], characterizations of the head-normalizing and left-most-normalizing terms of $\lambda \mathrm{x}$ were obtained in terms of typability in $\mathcal{D}_{\omega}$.

The main result of this paper is that typability in system $\mathcal{E}$ serves to characterize the strongly-normalizing terms of $\lambda \mathrm{x}$, and therefore that the rules (drop) and (K-cut) capture this important aspect of reduction in explicit substitutions calculi. But a natural question to raise at this point is whether rules (drop) and (K-cut) behave well in the presence of a universal type. In particular, we may ask whether the normalization theorems of [20] still hold in the presence of the new rules. In this section we show that this is the case. That is, we will verify that the $\mathcal{D}_{\omega}$-characterizations of normalizing and head-normalizing terms from [20] generalize in a natural way to $\mathcal{E}_{\omega}$. The first observation is that when a universal type is added to $\mathcal{E}$ the resulting system is equivalent to $\mathcal{D}_{\omega}$.

\subsection{Extending the type system}

Definition 7.1 The type system $\mathcal{E}_{\omega}$ is obtained from system $\mathcal{E}$ by adding the type constant $\omega$ and the rule:

$$
(\omega \mathrm{l}): \overline{\Gamma \vdash M: \omega}
$$

The type system $\mathcal{D}_{\omega}$ is obtained by adding $\omega$ and rule $(\omega \mathrm{l})$ to the system $\mathcal{D}$ of [20].

Theorem 7.2 Suppose $\Gamma \vdash M: \tau$ in system $\mathcal{E}_{\omega}$. Then $\Gamma \vdash M: \tau$ in system $\mathcal{D}_{\omega}$ as well.

Proof: By induction on the structure of derivations. In light of the equivalence between (drop) and (K-cut) it suffices to show that an application of rule (drop) can be simulated in $\mathcal{D}_{\omega}$. So 


$\stackrel{M \stackrel{h}{\longrightarrow} M^{\prime} M \text { not an abstraction }}{M N \stackrel{h}{\longrightarrow} M^{\prime} N} \stackrel{M \stackrel{h}{\longrightarrow} M^{\prime} M \text { not an abstraction }}{M\langle x=N\rangle \stackrel{h}{\longrightarrow} M^{\prime}\langle x=N\rangle}$
$\frac{M \stackrel{h}{\longrightarrow} M^{\prime}}{\lambda x \cdot M \stackrel{h}{\longrightarrow} \lambda x \cdot M^{\prime}}$

Figure 4: Head reduction

suppose

$$
\text { (drop) : } \frac{\Gamma \vdash M: \tau \quad \Delta \vdash N: \sigma}{\Gamma \vdash M\langle x=N\rangle: \tau}(x \notin a v(M))
$$

By induction we can derive $\Gamma \vdash M: \tau$ in $\mathcal{D}_{\omega}$, so certainly, using a $\mathcal{D}_{\omega}$-variant of Lemma 4.7(iv), $\Gamma,(x: \omega) \vdash M: \tau$. By $(\omega \mathrm{l}), \Gamma \vdash N: \omega$ in $\mathcal{D}_{\omega}$, so we have

$$
\text { (cut) }: \frac{\Gamma,(x: \omega) \vdash M: \tau \quad \overline{\Gamma \vdash N: \omega}}{\Gamma \vdash M\langle x=N\rangle: \tau}
$$

in $\mathcal{D}_{\omega}$, as desired.

\subsection{Head reduction and left-most reduction}

The head and left-most redexes from LC appear in $\lambda \mathrm{x}_{\mathrm{gc}}$ as head or left-most B-redexes. But the general notion of head or left-most redex in $\lambda \mathrm{x}_{\mathrm{gc}}$ must take the rules for applying substitutions into account. In fact, the correct definitions of head and left-most reduction are more subtle than in LC. Essentially this is because $\lambda \mathrm{x}_{\mathrm{gc}}$ has a critical pair, due to the following overlapping reductions:

$$
(\lambda x . M)\langle y=L\rangle N\langle y=L\rangle \longleftarrow((\lambda x \cdot M) N)\langle y=L\rangle \longrightarrow M\langle x=N\rangle\langle y=L\rangle
$$

Both these reductions could considered a "head reduction." In fact, it is our choice to consider them each to be head reductions.

Definition 7.3 (HeAd Reduction) Head reduction is the closure of the rules of $\lambda \mathrm{x}_{\mathrm{gc}}$ (Definition 2.4) under the structural rules of Figure 4. A term $M$ is head normalizing if there is no infinite head-reduction starting from $M$. The set of head normalizing terms is denoted $\mathcal{H} N$.

Definition 7.4 (LEFT-MOST REDUCTION) Left-most reduction is the closure of the rules of $\lambda \mathrm{x}_{\mathrm{gc}}$ under the structural rules in Figure 5. A term $M$ is left-most normalizing if there is no infinite left-most reduction starting from $M$. The set of left-most-normalizing terms is denoted $\mathcal{L} N$.

Observe that, in contrast to the classical notions, both head reduction and left-most reduction are non-deterministic strategies. Indeed, each of the reductions out of the critical pair noted earlier count as head reductions.

For example, let $T$ be $((\lambda x . M) N)\langle y=L\rangle$. Then $T$ can rewrite by left-most reduction either to $P \equiv M\langle x=N\rangle\langle y=L\rangle$, or (in two steps) to $Q \equiv((\lambda x . M\langle y=L\rangle) N\langle y=L\rangle)$. Then, since $\lambda x \cdot M\langle y=L\rangle$ is an abstraction, $Q$ left-most rewrites via rule $\mathrm{B}$ to $Q^{\prime} \equiv M\langle y=L\rangle\langle x=N\langle y=L\rangle\rangle$. 


$$
\begin{aligned}
& \frac{M \stackrel{l}{\longrightarrow} M^{\prime} \quad M \text { not an abstraction }}{M N \stackrel{l}{\longrightarrow} M^{\prime} N} \quad \frac{M \stackrel{l}{\longrightarrow} M^{\prime} M \text { not an abstraction }}{M\langle x=N\rangle \stackrel{l}{\longrightarrow} M^{\prime}\langle x=N\rangle} \\
& \underset{\lambda x . M \stackrel{l}{\longrightarrow} \lambda x . M^{\prime}}{\stackrel{l}{\longrightarrow}} \quad \frac{M_{i} \stackrel{l}{\longrightarrow} M_{i}^{\prime} M_{i} \text { left-most non-normal }}{x M_{1} \ldots M_{i} \ldots M_{n} \stackrel{l}{\longrightarrow} x M_{1} \ldots M_{i}^{\prime} \ldots M_{n}}
\end{aligned}
$$

Figure 5: Left-most reduction

\subsection{Characterization theorems}

We will assume familiarity with [20] in this subsection; we derive the characterization theorems by indicating how to lift the results of that paper. There is a technical issue to be dealt with, however: the garbage collection rule (gc) in the current paper is more liberal than the traditional rule in the system of [20]. In this section we refer to the traditional garbage collection rule as $\mathrm{gc}^{-}$:

$$
M\langle x=N\rangle \quad \longrightarrow \quad M \text {, if } x \notin f v(M) \quad\left(\mathrm{gc}^{-}\right)
$$

Formally, since [20] treats a different reduction system, it is difficult to quote results there in support of results about the system of this paper. But the arguments of the first paper carry over almost word-for-word. In light of this we have chosen to indicate below precisely where the distinction between the systems makes a difference, rather than repeating the entire development.

The following definitions are due to Cardone and Coppo [13]: A type is proper if it has no positive occurrence of $\omega$. A type is trivial if it can be generated by the following rules:

i) $\omega$ is trivial,

ii) If $\sigma$ is trivial and $\tau$ is any type, then $\tau \rightarrow \sigma$ is trivial,

iii) If $\sigma$ and $\tau$ are trivial, then $\sigma \cap \tau$ is trivial.

The following lemma isolates the place where we must acknowledge the difference in garbage collection rules.

Lemma 7.5 If $M$ is typable with a non-trivial type in system $\mathcal{D}_{\omega}$ then $M$ is head-normalizing in the calculus $\lambda \mathrm{x}_{\mathrm{gc}}$.

If $M$ is typable in system $\mathcal{D}_{\omega}$ with a type not involving $\omega$ then $M$ is left-most-normalizing in the calculus $\lambda \mathrm{x}_{\mathrm{gc}}$.

Proof: Each of these assertions is proved in [20] for the system $\lambda \mathrm{x}_{\mathrm{gc}^{-}}$(Theorems 8.1 and 8.2 there). We invite the reader to check that in that paper, the only places where the garbage collection rule is analyzed are Lemmas 3.2 and 3.5 and that the proofs of each of these Lemmas are essentially unchanged if the current, more liberal, gc rule is used. The rest of the development in [20] is unchanged, completing the proof.

Theorem 7.6 Let $M$ be a closed term. The following are equivalent.

i) $M$ is typable with a non-trivial type in system $\mathcal{E}_{\omega}$.

ii) $M$ is head-normalizing in the calculus $\lambda \mathrm{x}_{\mathrm{gc}}$.

iii) $M$ is head-normalizing in the calculus $\lambda \mathrm{x}$ (without garbage collection).

iv) $M$ has a head normal form.

v) $M$ is solvable, that is, there is an $n$ and terms $X_{1}, \ldots X_{n}$ such that $M X_{1} \cdots X_{n}=\lambda x . x$. 
Proof: By Theorem 7.2 we may replace, in $(i)$, " $\mathcal{E}_{\omega}$ " by " $\mathcal{D}_{\omega}$. ." Then each of the equivalences has been proved in [20] with the exception of the implication from (i) to (ii) since, in [20] garbage collection refers to the more restricted rule $\mathrm{gc}^{-}$. But for this implication we use Lemma 7.5 here.

Theorem 7.7 Let $M$ be a closed term. The following are equivalent.

i) $M$ is typable in system $\mathcal{E}_{\omega}$ with a type not involving $\omega$.

ii) $M$ is typable with a proper type in system $\mathcal{E}_{\omega}$.

iii) $M$ is left-most-normalizing in the calculus $\lambda \mathrm{x}_{\mathrm{gc}}$.

iv) $M$ is left-most-normalizing in the calculus $\lambda \mathrm{x}$ (without garbage collection).

v) $M$ has a normal form.

Proof: As for Theorem 7.6.

In Theorem 7.7, the implications (v) to (iii) and (v) to (iv) state that in $\lambda \mathrm{x}$ and $\lambda \mathrm{x}_{\mathrm{gc}}$ left-most reduction is a normalizing strategy.

\section{Conclusion}

We have defined an improved system of intersection types for calculi of explicit substitutions and shown that it characterizes the strongly normalizing terms. The new rules allowing us to type all strongly normalizing terms are consistent with the addition of a universal type, in the sense that the characterizations of head- and left-most-normalizing terms obtained in previous work are still valid in the extended system.

The new notion of available variable occurrence plays an important role in the type system, and indeed allows us to define a more powerful notion of garbage collection than has appeared elsewhere in the explicit substitutions literature. We like to note the similarity between the reduction rule (gc) and the classical 'mark-and-sweep' algorithm for garbage collection. As a matter of fact the computation of the set of available variables of a term corresponds to the 'mark'-phase, while the reduction using only rule (gc) corresponds to the 'sweep'-phase. Notice that this is not true for the similar rules of $[11,20]$. We think that it could be interesting to investigate the use of the garbage collection based on availability of variables in the implementations of functional programming languages.

\section{Acknowledgements}

The authors are grateful to Norman Danner, Frédéric Lang, Simona Ronchi della Rocca, and Kristoffer Rose for many helpful discussions. Moreover they thank the referees of the conferences LATIN'02 and TCS'02, and of the present submission for helpful comments.

\section{References}

[1] M. Abadi, L. Cardelli, P.-L. Curien, and J.-J. Lévy. Explicit substitutions. Journal of Functional Programming, 1(4):375-416, 1991.

[2] R. Amadio and P.-L. Curien. Domains and lambda-calculi. Cambridge University Press, 1998.

[3] S. van Bakel. Complete restrictions of the intersection type discipline. Theoretical Computer Science, 102(1):135-163, 1992. 
[4] S. van Bakel. Intersection Type Assignment Systems. Theoretical Computer Science, 151(2):385-435, 1995.

[5] S. van Bakel and M. Dezani-Ciancaglini. Characterizing strong normalization for explicit substitutions. In S. Rajsbaum, editor, LATIN'02, volume 2286 of Lecture Notes in Computer Science, pages 356-370. Springer-Verlag, 2002.

[6] H.P. Barendregt. The Lambda-Calculus, its syntax and semantics. Studies in Logic and the Foundation of Mathematics. Elsevier Science Publishers B.V. (North-Holland), Amsterdam, 1984. Second edition.

[7] H.P. Barendregt. Lambda calculi with types. In S. Abramsky, D.M. Gabbay, and T.S.E. Maibaum, editors, Handbook of Logic in Computer Science, volume 2, chapter 2, pages 117-309. Oxford University Press, 1992.

[8] Z. Benaissa, D. Briaud, P. Lescanne, and J. Rouyer-Degli. $\lambda v$, a calculus of explicit substitutions which preserves strong normalisation. Journal of Functional Programming, 6(5):699-722, 1996.

[9] R. Bloo. Preservation of Termination for Explicit Substitution. PhD thesis, Technische Universiteit Eindhoven, 1997. IPA Dissertation Series 1997-05.

[10] R. Bloo and J. H. Geuvers. Explicit substitution: on the edge of strong normalization. Theoretical Computer Science, 211:375 - 395, 1999.

[11] R. Bloo and K. H. Rose. Preservation of strong normalisation in named lambda calculi with explicit substitution and garbage collection. In CSN'95, pages 62-72, 1995.

[12] N. G. de Bruijn. A namefree lambda calculus with facilities for internal definition of expressions and segments. TH-Report 78-WSK-03, Department of Mathematics, Technological University Eindhoven, Netherlands, 1978.

[13] F. Cardone and M. Coppo. Two extension of Curry's type inference system. In P. Odifreddi, editor, Logic and Computer Science, volume 31 of APIC Series, pages 19-75. Academic Press, New York, NY, 1990.

[14] M. Coppo and M. Dezani-Ciancaglini. A new type assignment for lambda-terms. Archiv für mathematische Logik und Grundlagenforschung, 19:139-156, 1978.

[15] M. Coppo and M. Dezani-Ciancaglini. An extension of the basic functionality theory for the $\lambda$-calculus. Notre-Dame Journal of Formal Logic, 21(4):685-693, 1980.

[16] N. Dershowitz and Z. Manna. Proving termination with multiset orderings. Communications of the ACM, 22(8):465-476, 1979.

[17] M. Dezani-Ciancaglini, F. Honsell, and Y. Motohama. Compositional characterization of lambdaterms using intersection types. In M. Nielsen and B. Rovan, editors, MFCS'00, volume 1893 of Lecture Notes in Computer Science, pages 304-314. Springer-Verlag, 2000.

[18] R. Di Cosmo and D. Kesner. Strong normalization of explicit substitutions via cut elimination in proof nets. In LICS'97, pages 35-46. IEEEC Society Press, 1997.

[19] D. Dougherty and P. Lescanne. Reductions, intersection types, and explicit substitutions (extended abstract). In S. Abramsky, editor, TLCA'01, volume 2044 of Lecture Notes in Computer Science, pages 121-135. Springer-Verlag, 2001.

[20] D. Dougherty and P. Lescanne. Reductions, intersection types, and explicit substitutions. Mathematical Structures in Computer Science, 13(1):55-85, 2003.

[21] A.G. Dragalin. Mathematical Intuitionism: Introduction to Proof Theory, volume 67 of Translations of Mathematical Monographs. American Mathematical Society, 1987.

[22] J. Gallier. Typing untyped lambda terms, or reducibility strikes again. Ann. Pure Appl. Logic, 91:231-270, 1998.

[23] S. Ghilezan. Strong normalization and typability with intersection types. Notre Dame Journal of Formal Logic, 37(1):44-52, 1996.

[24] J. Goubault-Larrecq. Lambda-calcul, logique et machines. École Normale Supérieure de Cachan, 2001.

[25] H. Herbelin. Explicit substitutions and reducibility. Journal of Logic and Computation, 11(3):429-449, 2001.

[26] F. Kamareddine and A. Ríos. Extending a lambda-calculus with explicit substitution which preserves strong normalisation into a confluent calculus on open terms. Journal of Functional Programming, 7(4):395-420, 1997.

[27] J.-L. Krivine. Lambda-calcul, types et modèles. Masson, Paris, 1990. 
[28] J.-L. Krivine. Lambda calculus, types and models. Ellis Horwood, 1993.

[29] D. Leivant. Typing and computational properties of lambda expressions. Theoretical Computer Science, 44(1):51-68, 1986.

[30] S. Lengrand, D. Dougherty, and P. Lescanne. An improved system of intersection types for explicit substitutions. In Conference on Theoretical Computer Science, IFIP Congress, pages 511-524. Kluwer Academic Publishers, 2002.

[31] P. Lescanne. From $\lambda \sigma$ to $\lambda v$ : a journey through calculi of explicit substitutions. In Hans-J. Bôhm, editor, POPL'94, pages 60-69. ACM Press, 1994.

[32] P. Lescanne and J. Rouyer-Degli. The calculus of explicit substitutions $\lambda v$. Technical Report RR2222, INRIA-Lorraine, January 1994.

[33] P.-A. Melliès. Typed $\lambda$-calculi with explicit substitution may not terminate. In M. Dezani and G. Plotkin, editors, TLCA'95, volume 902 of Lecture Notes in Computer Science, pages 328-334. SpringerVerlag, 1995.

[34] J.C. Mitchell. Type systems for programming languages. In J. van Leeuwen, editor, Handbook of Theoretical Computer Science, volume B, pages 415-431. Elsevier Science Publishers B.V. (NorthHolland), Amsterdam, 1990.

[35] J.C. Mitchell. Foundation for Programming Languages. MIT Press, 1996.

[36] G. Pottinger. A type assignment for the strongly normalizable $\lambda$-terms. In J.P. Seldin and J.R. Hindley, editors, To H.B. Curry: Essays on Combinatory Logic, Lambda Calculus and Formalism, pages 561-578. Academic Press, 1980.

[37] E. Ritter. Characterising explicit substitutions which preserve termination. In J.-Y. Girard, editor, TLCA'99, volume 1581 of Lecture Notes in Computer Science, pages 325-339. Springer-Verlag, 1999.

[38] K.H. Rose. Operational Reduction Models for Functional Programming Languages. PhD thesis, DIKU, Universitetsparken 1, DK-2100 København Ø, February 1996. DIKU report 96/1.

[39] P. Sallé. Une extension de la théorie des types en $\lambda$-calcul. In G. Ausiello and C. Böhm, editors, ICALP'78, volume 62 of Lecture Notes in Computer Science, pages 398-410. Springer-Verlag, 1978.

[40] W.W. Tait. Intensional interpretations of functionals of finite type I. Journal of Symbolic Logic, 32:198-212, 1967. 